\title{
V. Veröffentlichungen von Sternwarten und Instituten
}

Die unten genannten Sternwarten sind in dem Verzeichnis der Veröffentlichungen von Sternwarten und Instituten unter dem Ort (rechte Spalte dieser Tabelle) aufgeführt.

\begin{tabular}{|c|c|}
\hline Nameder Sternwarte & Ort \\
\hline $\begin{array}{l}\text { Agassiz Station } \\
\text { Allegheny Observatory } \\
\text { Carter Observatory } \\
\text { Commonwealth Scientific and Industrial Research } \\
\text { Organization, Australia } \\
\text { Dearborn Observatory of Northwestern University } \\
\text { Dominion Observatory } \\
\text { Dominion Astrophysical Observatory } \\
\text { David Dunlap Observatory, University of Toronto } \\
\text { Dunsink Observatory } \\
\text { Observatorio del Ebro } \\
\text { Engelhardt Observatorium } \\
\text { Observatorio Fabra } \\
\text { Flower Astronomical Observatory } \\
\text { Harvard College Observatory } \\
\text { Kapteyn Astronomical Laboratory } \\
\text { Lick Observatory } \\
\text { Goethe Link Observatory of Indiana University } \\
\text { Lowell Observatory } \\
\text { Leander McCormick Observatory of the University } \\
\text { of Virginia } \\
\text { McDonald Observatory } \\
\text { McMath-Hulbert Observatory } \\
\text { Observatory of the University of Michigan } \\
\text { Astronomical Observatory, University of Minnesota } \\
\text { Perkins Observatory } \\
\text { Radcliffe Observatory } \\
\text { Smithsonian Institution } \\
\text { Sproul Observatory } \\
\text { Staatliches Astronomisches Sternberg-Institut } \\
\text { Union Observatory } \\
\text { Vassar College Observatory } \\
\text { Warner and Swasey Observatory } \\
\text { Washburn Observatory of the University of Wisconsin } \\
\text { Astronomical Observatory of Yale University } \\
\text { Yerkes Observatory }\end{array}$ & $\begin{array}{l}\text { Oak Ridge } \\
\text { Pittsburgh } \\
\text { Wellington, N. Z. } \\
\text { Sydney } \\
\text { Evanston, Ill. } \\
\text { Ottawa } \\
\text { Victoria, Canada } \\
\text { Richmond Hill } \\
\text { Dublin } \\
\text { Tortosa } \\
\text { Kasan } \\
\text { Barcelona } \\
\text { Upper Darby, Penn. } \\
\text { Cambridge, Mass. } \\
\text { Groningen } \\
\text { Mount Hamilton } \\
\text { Bloomington } \\
\text { Flagstaff, Ariz. } \\
\text { Charlottesville } \\
\text { Fort Davis, Tex. } \\
\text { Lake Angelus, } \\
\text { Pontiac, Mich. } \\
\text { Ann Arbor } \\
\text { Minneapolis } \\
\text { Delaware, Ohio } \\
\text { Pretoria } \\
\text { Washington } \\
\text { Swarthmore, Penn. } \\
\text { Moskau } \\
\text { Johannesburg } \\
\text { Poughkeepsie, N. Y. } \\
\text { Cleveland, Ohio } \\
\text { Madison } \\
\text { New Haven, Conn. } \\
\text { Williams Bay, Wis. }\end{array}$ \\
\hline
\end{tabular}




\section{Amsterdam}

114. Circular of the Astronomical Institute of the University of Amsterdam.

Nr. 2: A. Pannekoek, Periodicities in lunar eclipses. Ref. 3213.

3: D. Koelbloed, W. Veltman, On the electron temperature of the chromosphere and prominences. Ref. 6618.

\section{Ann Arbor}

115. Publications of the Observatory of the University of Michigan.

10: The structure of the galaxy. A symposium held in connection with the dedication of the Heber Doust Curtis Memorial Telescope, June 22-24, 1950. Ref. 12331.

J. Stebbins, Heber Curtis and the Michigan telescope. Ref.1052.

W. Baade, Galaxies - present day problems. Ref. 12402 .

N. U. Mayall, Comparison of rotational motions observed in the spirals M31 and M33 and in the galaxy. Ref. 12424.

R. Minkowski, Galactic distribution of planetary nebulae and Be stars. Ref. 12213.

W. W. Morga n, Application of the principle of natural groups to the classification of stellar spectra. Ref. 9538.

J. J. Nassau, W. W. Morgan, Distribution of early type stars of high luminosity near the galactic equator. Ref. 12320 .

F. D. Miller, Utilization of faint star counts at intermediate latitudes. Ref. 12319.

B. Lindblad, Dynamical interpretation of velocity distribution and structural details in the galaxy. Ref. 12342.

A. N. Vyssotsky, Some features of galactic structure revealed by stellar motions. Ref. 12358.

S. W. McCuskey, Recent investigations concerning the stellar luminosity function. Ref. 9718.

K. G. Henize, F. D. Miller, H-Alpha emission objects in the Magellanic clouds. Ref. 12417.

H. Shapley, Comparison of the Magellanic clouds with the galactic system. Ref. 12439.

\section{Århus}

116. Meddelelser fra Ole Romer-Observatoriet i Arhus.

Nr. 21: A. V. Nielsen, On two bright meteors. Vgl. AJB 50 Ref. 8278.

22: A. V. Nielsen, H. C. Schumacher and the Observatory at Altona during the war of 1848-50. Ref. 1039.

\section{Armagh}

117. Contributions from the Armagh Observatory.

Nr. 3: E. J. Ôpik, Stellar models with variable composition. II. Sequences of models with energy generation proportional to the fifteenth power of temperature. Ref. 5225.

4: E. M. Lindsay, The distribution of stars brighter than photographic magnitude 14,0 between galactic longitudes $290^{\circ}$ and $360^{\circ}$ and within galactic latitudes $\pm 30^{\circ}$. Ref. 12315 .

5: Vgl. AJB 50 Ref. 5224.

6: E. J. O pik, Collision probabilities with the planets and the distribution of interplanetary matter. Ref. 7018.

7: Vgl. AJB 50 Ref. 5225.

8: E. J. Öpik, Rotational currents. Ref. 4310. 
XVIII Übersicht über die referierten Zeitschriften

118. Armagh Observatory Leaflet.

Nr. 7: E. M. Lindsay, Report on Armagh Observatory for the year 1950. Ref. 1101.

8: E. B. Armstrong, Opposition of Mars, 1950. Ref. 7402.

9: E. J. Opik, Astronomy and the bottom of the sea. Ref. 8322 .

10: S. Grew, Meteorology at Armagh Observatory : General notes and a study of rainfall. Ref. 1202.

\section{Asiage}

119. Contributi dell'Osservatorio Astrofisico dell'Università di Padova in Asiago.

Nr. 20: G. de Strobel, Velocità radiali di $\zeta_{1}$ Ursae majoris e nuovi elementi dell'orbita. Ref. 10213.

21: L. Rosino, Ricerche sugli ammassi globulari. V. Diagramma colore-grandezza e distanza dell'ammasso globulare M 56. Ref. 12128.

22: G. de Strobe1, Grandezze fotovisuali della variabile BL Casgiopeiae. Ref. 11229.

\section{Athen}

120. National Observatory of Athens - Greece. Bulletin of the Astronomical Institute. Year: 1951.

Nr. 1-2: Vgl. § 68, 71, 73, 75, 76, 77, 112.

\section{Bamberg}

121. Kleine Veröffentlichungen der Remeis-Sternwarte.

Nr. 6: H.-U. Sandig, Die Verbesserung der Sternörter für eine Neureduktion älterer Azimut- und Längenbestimmungen. Vgl. AJB 50 Ref. 9111.

Basel

122. Veröffentlichungen der Astronomisch-Meteorologischen Anstalt der Universität Basel.

Nr. 1: J. O. Fleckenstein, Die Struve-Niethammersche Methode der Vertikalpassagen zur Bestimmung von Polhöhenschwankungen. Ref. 3111.

\section{Belgrad}

123. Publications de l'Observatoire Astronomique de Belgrade.

Nr. 4: P. M. Djurković, B. M. Ševarlič, Z. M. Brkić, Détermination de latitude de l'Observatoire Astronomique de Belgrade, 1947. Ref. 13206.

124. Publications de l'Observatoire Astronomique de Belgrade. Bulletin.

15: B. Popović, Rapport annuel sur l'état et l'activité de l'Observatoire Astronomique de Belgrade en 1950. Ref. 1101.

Z. M. Brki ć, P. M. Djurkovið, B. M. Sevarlić, Observations méridiennes, faites à l'instrument de passage $($ de $100 \mathrm{~mm}) \mathrm{du}$ Service de l'heure en 1950. Ref. 2854.

M. Simić, M. Protitch, Activité des taches solaires en 1950. Ref. 6353.

M. Protitch, L'éclipse totale de la lune, le $2 / 3$ avril 1950, observé à Belgrade. Ref. 7333. 
B. M. Sevarlić, Observations de l'éclipse totale de lune du 26 septembre 1950. Ref. 7334.

Observations des occultations en 1950. Ref. 7341.

Predictions for 1951 of occultations of stars by the moon visible at Belgrade. Ref. 7340.

M. Protitch, Observations photographiques des Petites Planètes, faites à l'astrographe de $160 \mathrm{~mm}$. Ref. 7562 .

Z. M. Brkič, P. M. Djurkovič, B. M. Ševarlić, Observations à la lunette zénithale (de $110 \mathrm{~mm}$ ) du Service de latitude de l'Observatoire en 1950. Ref. 13202.

B. M. Ševarlič, Z. M. Brki ć, Observations faites avec l'instrument universel. Ref. 13242.

\section{Bergedorf}

Siehe unter Hamburg.

\section{Berkeley}

125. Berkeley Reprint.

Nr. 1: Vgl. AJB 50 Ref. 9555 (12 Lac).

2: Nicht eingegangen.

3: O. Struve, A puzzling stellar spectrum (17 Leporis). Ref. 9568.

4-9: Nicht eingegangen.

10: O. Struve, Notes on stellar spectra. Ref. 9550.

11: M. F. Walker, Variability of $v$ Eridani. Ref. 11229. J. G. Phillips, Temperatures of late-type stars from the band spectra. Ref. 9603.

12: N. E. Gould, The dark lanes near $\rho$ Ophiuchi. Ref. 12513.

D. H. McNamara, R. D. Levée, Changes in the spectrum of $\sigma$ Scorpii. Ref. 9568.

0 . Struve, Some comments on $\beta$ Canis majoris and related stars. Ref. 9551.

R. D. Levée, Overlapping Balmer lines in $\alpha$ Lyrae. Ref. 9568.

13: D. H. McNamara, The eclipsing binary U Sagittae. Ref. 11119.

14: D. Layzer, The scattering of slow electrons by atoms. Ref. 234.

\section{Berlin}

126. Berliner Astronomisches Jahrbuch für 1953. 178. Jahrgang. Herausgeber: Astronomisches Recheninstitut der Deutschen Akademie der Wissenschaften zu Berlin und Astronomisches Rechen-Institut zu Heidelberg. Ref. 401.

127. Mitteilungen aus dem Astronomischen Recheninstitut der Deutschen Akademie der Wissenschaften zu Berlin.

E. E. Kühne, Ubber die mit den Methoden der speziellen Störungen erreichbare Genauigkeit. Ref. 4212.

A. Kahrstedt, Die Genauigkeit numerischer Integrationen von speziellen Störungen über längere Zeiträume. Ref. 4211.

128. Mitteilungen der Archenhold-Sternwarte Berlin-Treptow.

Nr. 16: W. Neumann, Auswertungen von Schattenantrittsbeobachtungen während der totalen Mondfinsternis 2. April 1950. Ref. 7833. M. Schubert, Die Silberkugel-Photometrie und ihre Anwendung auf die Mondfinsternis vom 2./3. April 1950. Ref. 7333.

G. Schirdewahn, Die Marsopposition 1950. Ref. 7402.

E. Rohde, G. Schirdewahn, Saturn 1950. Ref. 7702.

17: D. Wattenberg, Die Photographie des Himmels. Ref. 2263. 
18: D. Wattenberg, Die Photographie der Sonne. Ref. 2264.

19: D. Wattenberg, Entwicklung und Wirken der ArchenholdSternwarte im Jahre 1950. Ref. 1101.

20: I. Nickel, G. Schirdewahn, Bericht über die Untersuchung von Sonnenbeobachtungen 1950. Ref. 6353.

21: D. Wattenberg, Zur Definition des Planetenbegriffs. Ref. 7027.

22: E. Mädlow, Periodizität der Oberflächengebilde auf Jupiter. Ref. 7607.

23: D. Wa ttenberg, Planetenähnliche Fixsternbegleiter. Ref.10012. 128a. Astronomischer Nachrichtendienst der Archenhold-Sternwarte zu BerlinTreptow. 3. Jahrgang Nr. 1-24.

Bern

129. Veröffentlichungen des Astronomischen Instituts der Universität Bern.

Nr. 8: R. Kurth, Die Masse der Kugelsternhaufen. Ref. 12122.

9: R. Kurth, Die Entwicklung der Kugelsternhaufen. Ref. 12123.

\section{Bjurakan}

130. Mitteilungen (Soobschtschenija) des Observatoriums Bjurakan.

Nr. 6: W. A. Ambarzumjan, Zur Theorie der Schwankungen in der scheinbaren Verteilung der Sterne am Himmel. Ref. 4401.

7: L. W. Mirsojan, Spektralphotometrische Untersuchung einer Reihe von Sternen früher Spektralklassen. Ref. 9536.

8: G. S. Badaljan, Die Farbenindizes der langperiodischen Cepheiden. Ref. 11143.

9: B. E. Markarjan, Über die Klassifikation der offenen (galaktischen) Sternhaufen. II. Vorläufiges Verzeichnis offener O-Haufen. Ref. 12109.

\section{Bloomington}

131. Publications of the Goethe Link Observatory of Indiana University.

Nr. 3: Vgl. AJB 49 Ref. 9502.

4: Vgl. AJB 52.

5: Vgl. AJB 52.

6: J. Cuffey, Linearity in photoelectric equipment. Ref. 2213.

132. Goethe Link Observatory of Indiana University. Reprint.

Nr. 4: J. B. Irwin, An astronomical visit to South Africa. Ref. 1252.

\section{Bologna}

133. Pubblicazioni dell'Osservatorio Astronomico Universitario di Bologna.

5 Nr. 14: P. Tempesti, Indice di colore del nucleo della nebulosa planetaria in A.R. $=23^{\mathrm{h}} 33^{\mathrm{m}} 24^{\mathrm{s}}$ e D $=+30^{\circ} 11^{\prime}(1950.0)$. Ref. 12224.

15: I. Rosino, Ricerche sugli ammassi globulari. VI. L'ammasso globulare di Baade in A.R. $15^{\mathrm{b}} 13^{\mathrm{m}} 30^{\mathrm{s}} \mathrm{e} \mathrm{D}+0^{\circ} 4^{\prime}(1950.0)$. Ref. 12127.

16: L. Rosino, Alcuni aspetti delle ricerche sulle nebulose planetarie. Ref. 12216.

\section{Bonn}

134. Veröffentlichungen der Universitäts-Sternwarte zu Bonn.

Nr. 37: J. Meurers, Die Feinstruktur der scheinbaren Sternverteilung. Ref. 12317. 
38: K. Terheyden, Nomogramme zur Lösung astronomischer Aufgaben. Zweite Folge. Zur geographischen Ortsbestimmung und astronomischen Navigation. Ref. 3017.

\section{Bordeaux}

135. Annales de l'Observatoire de Bordeaux.

18 2: J. Dubois, Contribution à l'étude de l'ombre de la terre. Ref. 7325.

136. Observatoire de l'Université de Bordeaux (Floirac). Série A.

Nr. 2: Dumas, Parcelier, Chronographe imprimant à roue phonique. Ref. 2313.

3: P. Sémirot, Principes d'un nouvel instrument des passages. Ref. 2132.

4: P. Parcelier, Nouveau relais électronique du service méridien. Ref. 2236.

137. Publications de l'Observatoire de Bordeaux. Nouvelle Série.

Nr. 8: R. Mercier. Vgl. AJB 50 Ref, 10109.

P. Sémirot, Principes d'un nouvel instrument des passages. Ref. 2134.

Chopinet, P. Sémirot. Vgl. AJB 50 Ref. 9203.

Chopinet, Dupouy, Teulet, Positions photographiques de Némausa. Ref. 7562.

Dubois, Mercier, Mesures d'étoiles doubles au micromètre à double image de Muller. Ref. 10125.

9: P. Sémirot, Principes d'un nouvel instrument des passages. Ref, 2133.

10: Vgl. AJB 50 Ref. 9208.

\section{Breslau}

Siehe unter Wroclaw.

\section{Brno}

138. Contributions from the Astronomical Institute of the Masaryk University Brno, Czechoslovakia.

1 8: L. Perek, Distribution of mass in the galaxy and the densities of RR Lyrae variables. Ref. 12845.

\section{Brooklyn}

139. The Goethe Link Observatory. Brooklyn, Indiana. Reprint.

Nr. 1: V. E. Maier, The new Goethe Link Observatory. Vgl. AJB 42 Ref. 406.

2: J. Cuffey, The galactic clusters. Vgl. AJB 43 Ref. 8705.

3: F.K. Edmondson, Recent developments at the Goethe Link Observatory. Vgl. AJB 48 Ref. 1208.

\section{Bruxelles}

140. Université de Bruxelles. Institut d'Astronomie.

(1) Nr. 11: L. Evrard, Sur l'équilibre relatif des fluides hétérogènes en rotation. Le problème des étoiles doubles. Ref. 4304.

141. Université de Bruxelles. Institut d'Astronomie.

(2) Nr. 61: J. F. Cox, Notice sur Albert Lancaster. Ref. 1018. 
62: J. F. Cox, S. D. Téhérany, Sur des observations effectuées à l'Université de Bruxelles pour y constituer une station de Laplace. Ref. 13204.

63: A. Huaux, Sur des déplacements concomitants de l'axe instantané de rotation et de l'axe principal instantané d'inertie de la terre. Ref. 13115.

64: A. Huaux, Sur les déplacements concomitants de l'axe instantané de rotation et de l'axe principal instantané d'inertie de la terre. II. Ref. 13116.

65: F. H. van den Dungen, J. F. Cox, J. van Mieghem, Rocherche d'effets de sélection attribuables aux fluctuations de la rotation du globe terrestre. Ref. 13110.

66: J. F. Cox, The doubly equidistant projection. Ref. 13004.

67: A. Koeckelenbergh, Sur un modèle de chromosphère solaire en équilibre hydrostatique. Ref. 6617.

68: F. H. van den Dungen, J. F. Cox, J. van Mieghem, Sur les fluctuations saisonnières de la rotation du globe terrestre et la compensation des échanges de quantités de mouvement entre l'atmosphère et le globe. Ref. 13111.

69: J. F. Cox, A propos du centenaire de l'expérience du pendule de Foucault. Ref. 1409.

70: A. Huaux, Sur un modèle de satellite en glace. Ref. 5213.

71: J. F. Cox, Vers une nouvelle définition de l'échelle des temps. Ref. 13109.

72: E. Schatzman, Sur la stabilité de certains modèles de planètes. Ref. 5231.

\section{Budapest-Szabadsághegy}

142. Mitteilungen der Sternwarte der ungarischen Akademie der Wissenschaften.

Nr. 24: I. Guman, Das photometrische Doppelsternsystem CD Vulpeculae. Ref. 11110.

25 : I. K. Csada, On the origin of the magnetic field of the sunspots. Ref. 6308.

26: I. K. Csada, On the magnetic effects of turbulence in ionized gases. Ref. 209.

Cambridge, Engl.

143. Contributions from the Cambridge Observatories.

Nr. 5: E. H. Linfoot, Error balancing in fast Schmidt cameras. Ref. 2014.

6: H. von Klüber, An attempt to detect a general magnetic field of the sun by a spectrographic method, using a Lummer plate. Ref. 6125.

7: P. Na ur, Computation of special perturbations by an electronic calculator. Ref. 4218.

8: F. Schmeidler, Proper-motion distribution and mean parallaxes of the stars of the General Catalogue in different galactic latitudes and longitudes. Ref. 12347.

9: P. B. Fellgett, An exploration of infra-red stellar magnitudes using the photo-conductivity of lead sulphide. Ref. 2219.

144. Cambrid ge Observatories Reprints.

Nr. 1: A. Beer, M. W. Ovenden, Zeta Aurigae: Photoelectric observations of the partial phase at egress on September 20, 1950. Ref. 11101. 
2: University of Cambridge. Report of the Observatories Syndicate for the year ending 1951 September 30. Ref. 1101.

Cambridge, Mass.

145. Harvard College Observatory Circular.

Nr. 456: M. G. Fracastoro, A spectrophotometric study of Zeta Aurigae. Ref. 11105.

457: Z. Kopal, C. G. Treuenfels, The effective temperatures of components of eclipsing binary systems. Ref. 9602 .

146. Harvard Reprint.

Nr. 342: D. Hoffleit, The discovery and exploitation of spectroscopic parallaxes. Ref. 9301.

343: F. L. Whipple, The theory of micro-meteorites. Part I. In an isothermal atmosphere. Part II. In heterothermal atmospheres. Ref. 8335.

344: H. Shapley, Proxima Centauri as a flare star. Ref. 11186.

345: H. Shapley, Magellanic clouds. I. Transparency. Ref. 12435.

346: H. Shapley, V. McKibben Nail, Magellanic clouds. II. Supergiant red variable stars in the small cloud. Ref. 12436.

347: H. Shapley, Galactic and extragalactic studies. XXI. Distribution of 95.000 galaxies in the north galactic cap. Ref. 12437.

348: J. W. Warwick, Curve of growth polarisation. Ref. 9813.

349: F. L. Whipple, A comet model. II. Physical relations for comets and meteors. Ref. 8026.

350: D. H. Menzel, H. K. Sen, Transfer of radiation. II. Radiative transfer in absorption lines. Ref. 5111.

351: D. H. Menzel, H. K. Sen, Transfer of radiation. III. Reflection effect in eclipsing binaries. Ref, 5112.

352: H. Shapley, A survey of the inner metagalaxy. Ref. 12438.

853: W. J. Luyten, Forty-four new white dwarfs in the southern hemisphere. Ref. 9716.

354: Y. O hman, Some observations of dark filaments in prominences. Ref. 6631.

355: R. N. Thomas, F. L. Whipple, The physical theory of meteors. II. Astroballistic heat transfer. Ref. 8252 .

856 : M. W. Ma y all, Variable star notes from the American Association of Variable Star Observers in 1951. Ref. 11229.

857: Vgl. AJB 52.

358: G. C. McVittie, C. Payne-Gaposchkin, A model of a spiral galaxy. Ref. 12426.

147. Harvard Reprint Series II.

Nr. 36: H. Shapley, V. McKibben Nail, NGC 1866 and the Magellanic cloud variables. Ref. 12112.

37: H. Shapley, Comparison of the Magellanic clouds with the galactic system. Ref. 12439.

38: F. W. Wright, The photographic Leonid meteors. Ref. 8284.

39: P. Carroll, R. E. MoCrosky, R. C. Wells, F. L. Whipple, Film molding and photographic accessories for the super-Schmidt meteor cameras. Ref. 2257.

148. Harvard College Observatory Bulletin.

Nr. 920: B. J. Bok, Irregularities in the galactic absorbing layer. Ref. 12503.

U. van Wijk, The applicability of the virial theorem to evolving clusters. Ref. 12116. 
P. F. Bok, Nova Zwicky and Nova Haro. § 113.

M. K. V. Bappu, On the light curves of RX Librae and TV Can is Major. Ref. 11229.

M. Olmsted, Observations of six Cepheid variables in Cygnus. Ref. 11229.

V. McKibben Nail, Three notes on variable stars in the small Magellanic cloud. Ref. 12425.

V. McKibben Nail, Variables in the globular cluster $47 \mathrm{Tu}$ canae. Ref. 12124.

I. King, New variables and periods in the globular cluster Messier 55. Ref. 12121.

D. Hoffleit, On the distribution of long-period variables implied by the period-luminosity relation. Ref. 11170 .

S. Gaposchkin, The eclipsing system RZ Eridani. Ref. 11229.

S. Gaposchkin, A new bright double-lined eclipsing variable $\left(\mathrm{BD}+26^{\circ} 2508\right)$. Ref. 11231 .

G. G. Cillie, B. J. Bok, The photoelectric light curve of TW Ceti. Ref. 11229.

D. Hoffleit, A reminiscence: Hertzsprung's peculiar object of short duration. Ref. 11171.

M. W. Mayall, New peculiar spectra. Ref. 9530.

H. Zirin, A list of peculiar A stars on objective prism plates. Ref. 9564.

I. King, HD 32633, a peculiar spectrum of type A. Ref. 9568.

R. J. Davis, Tables of the Secant of Zenith Distance. Ref. 3003. G. G. Cillié, The photoelectric light curve of YY Eridani. Ref. 11229.

D. Hoffleit, Three possible variable-emission spectra. Ref.11172.

149. Harvard College Observatory. The American Association of Variable Star Observers. Quarterly Report.

Nr. 3-8: M. W. Mayall, Observations of variable stars. Ref. 11220, § 113.

150. Harvard College Observatory. Annual Report.

Ref. 1101.

151. Harvard College Observatory. Announcement Card 1067 (1950), 1110-1163.

Camberra

152. Report of the Commonwealth Astronomer for the year 1950. Mit Supplement. Ref. 1101.

\section{Cape}

153. Annals of the Cape Observatory.

16: J. Jackson, Stellar parallaxes $\left(6^{\text {th }}, 7^{\text {th }}\right.$ and $8^{\text {th }}$ series) determined in the years 1937 1948 with the Victoria telescope of the Royal Observatory, Cape of Good Hope. Ref. 9306.

154. J. Jackson, Third Cape Catalogue of Stars for the Equinox of 1925.0. Catalogue of 6597 Stars between the Equator and Declination $-30^{\circ}$. Ref. 9103.

155. Report of His Majesty's Astronomer at the Cape of Good Hope to the Secretary of the Admiralty for the year 1950. Ref. 1101.

\section{Capodimonte}

Siehe unter Napoli. 


\section{Castel Gandolfo}

156. Specola Astronomica Vaticana. Ricerche Astronomiche.

2 10: W. J.Miller, Seven new eclipsing variables, VV 4-10. Ref. 11240.

11: R. Leclaire, Observations photographiques de Nemausa (51) pour l'opposition de 1950. Ref. 7562.

12: J. de Kort, W. J. Miller, The machine calculation of Greenwich mean astronomical time and universal time from local sideral time. Ref. 3118 .

13: J. G. E. G. Voûte, Observations of double stars. Ref. 10125.

14: G. Stein, Creazione senza creatore? Ref. 658.

\section{Catania}

157. Osservatorio Astrofisico - Catania. Contributi Astrofisici.

Nr. 70: E. de Caro, S. Grasso, Sull'orbita del sistema binario $\sigma$ Coronae borealis. Ref. 10128.

71: E. de Caro, E. Fichera, Nuova determinazione dell'estinzione atmosferica a Catania, in quattro regioni dello spettro visibile. Ref. 7255.

72: L. Taffara, L'attività del sole nell'anno 1950. Ref. 6353, 6660.

158. Osservatorio Astrofisico di Catania. Annuario 1952. Ref. 436.

\section{Charkow}

159. Publikationen (Trudy) des Astronomischen Observatoriums der GorkijStaatsuniversität Charkow.

1 (9): N. P. Barabaschew, Über die Verlängerung der Hörnerspitzen der Venussichel. Ref. 7115.

N. P. Barabaschew, Ǔber Wolken, Nebel und Niederschläge auf dem Mars. Ref. 7404.

N. P. Barabaschew, Ergebnisse der Photometrie des Saturn. Ref. 7703.

N.P. Barabaschew, A. T. Tschekirda, Photographische Spektralphotometrie von Venus, Mars, Jupiter und Saturn. Ref. 7116. A. T. Tschekirda, Die Helligkeitsverteilung auf der Jupiterscheibe im roten, gelben und grünen Licht. Ref. 7609.

G. M. Bashenow, Untersuchung der Konvergenz einer Reihe in der Theorie der Bahnbestimmung der Planeten und Kometen. Ref. 4501.

G. M. Bashenow, Vorausberechnung der größten gegenseitigen Annäherungen der Planeten. Ref. 7002.

K. N. Kusmenko, ťber die Teilungsfehler des Meridiankreises des Astronomischen Observatoriums Charkow. Ref. 2506.

G. M. Bashenow, Über die Bestimmung einer Kreisbahn. Ref. 4502.

G. R. Possoschkow, Rektaszensionsbeobachtungen von Jupiter am Passageninstrument Bamberg. Ref. 7601.

L. I. Schingarjow, Utber die Rolle der Lichtstreung in der Venusatmosphäre. Ref. 7124.

160. Zirkular des Astronomischen Observatoriums der Gorkij-Staatsuniversität Charkow.

Nr. 8: N.P. Barabaschew, Einige Ergebnisse der photometrischen Bearbeitung der 1933 und 1939 erhaltenen Marsaufnahmen durch Lichtfilter. Ref. 7403. 
R. M. Rapota, Die Aktivität der Photosphäre und Chromosphäre der Sonne vom 1. Juli bis 31. Dezember 1950 nach Beobachtungen am Astronomischen Observatorium Charkow. Ref. 6353, 6660.

\section{Cincinnati}

161. Minor Planet Circulars Nr. 490-708. Ref. 7562.

Coimbra

162. Anais do Observatório Astronómico da Universidade de Coimbra. Primeira Secção.

11 1939: Fenómenos solares. Ref. 6353, 6660.

163. Efemérides Astronómicas para o ano de 1952. Ref. 402.

\section{Córdoba}

164. Resultados del Observatorio Nacional Argentino.

38 1: Catálogo D. Ref. 9118.

165. Observatorio de Cordoba. Tirada Aparte.

Nr. 36: J. Sahade, A spectrographic study of the eclipsing star V 377 Centauri. Ref. 11129.

37-46: Vgl. AJB 50.

47: J. Bobone, Photographic observations of comets. $\$ 81$.

\section{Delaware}

166. Contributions from the Perkins Observatory.

Nr. 27: L. H. Aller, P. C. Keenan, The spectra of R Andromedae (maximum) and $\mu$ Cephei between 7400 and 8800 A. Ref. 11159.

28: J. Belzer, G. Gamow, G. Keller, On the stellar dynamics of spherical galaxies. Ref. 4402.

29: A. Slettebak, Lines of neutral oxygen in the infrared spectra of Be stars. Ref. 9542.

A. Slettebak, Lines of neutral oxygen in the infrared spectra of peculiar A stars. Ref. 9543.

30: P. C. Keenan, G. Keller, HD 26 - an unusual high-velocity star. Ref. 9524.

31: G. Keller, D. N. Limber, A photoelectric study of the eclipsing stars RS Canum Venaticorum and YY Sagittarii. Ref. 1111.

167. The Perkins Observatory. Miscellaneous Scientific Papers. Reprint.

Nr. 37: A. Offner, W. B. Decker, An f:1.0 camera for astronomical spectroscopy. Ref. 2127.

\section{Dablin}

168. Contributions from the Dunsink Observatory.

Nr. 1: H. E. Butler, The equivalent widths of Calcium and hydrogen absorption lines in the near infra-red of stellar spectra. Ref. 9517.

2: H. E. Butler, Photoelectric recording of stellar occultations. Ref. 3205.

169. Dunsink Observatory Reprints.

Nr. 2: H. E. Butler, Further recordings of stellar scintillation. Ref. 72145.

3: H. E. Butler, Photo-electric observations of the scintillations of stars. Ref. 72144.

4: H. E. Butler, Scintillation and atmospheric seeing. Ref. 72146. 


\section{Edinbargh}

170. Communication from the Royal Observatory, Edinburgh.

Nr. 2: R. Wilson, The blue sun of 1950 September. Ref. 6029.

Evanston, Ill.

171. Contributions from the Dearborn Observatory.

Nr. 1: K. A. Strand, The orbit and parallax of Procyon. Ref. 10120.

2: Vgl. AJB 49 Ref. 5119.

3: Vgl. AJB 49 Ref. 10125 (99 Her).

\section{Fort Davis}

172. Contributions from the McDonald Observatory.

Nr. 198: A. Brown, The color-magnitude array for stars in the globular cluster M 15 . Ref. 12119.

199: W. P. Bidelman, Spectral classification of stars listed in Miss Payne's catalogue of c stars. Ref. 9509.

200: W. A. Hiltner, The Wolf-Rayet spectroscopic binary $\mathrm{BD}+36^{\circ}$ 3991. Ref. 10203.

201: W. A. Hiltner, Polarization of stellar radiation. III. The polarization of 841 stars. Ref. 12542.

202: Vgl. AJB 52.

203: W. P. Bidelman, P. C. Keenan, The Ba II stars. Ref. 9510.

204: Vgl. AJB 62.

205: H. L. Johnson, W. W. Morgan, On the color-magnitude diagram of the Pleiades. Ref. 12108.

Freiburg i. Br.

173. Veröffentlichungen des Fraunhofer-Instituts Freiburg i. Br.

Sonnen-Zirkular für den Monat Oktober 1950-September 1951. $\$ 63,66,67,72,127$.

174. Mitteilungen aus dem Fraunhofer-Institut, Freiburg i. Br.

Nr. 1: A. Behr, Zur Entfernungsskala der extragalaktischen Nebel. Ref. 12404.

2: A. Behr, Ein lichtelektrisches Sternphotometer mit Sekundärelektronenvervielfacher. Ref. 2205.

3: A. Behr, Die Intensitätsvariation der grünen Koronalinie 1943 -1950. Ref. 6702.

4-7: Vgl. AJB 52.

8: K. O. Kiepenheuer, Über die Verdampfung der Sonnenkorona. Ref. 6708.

\section{Genf}

175. Publications de l'Observatoire de Genève.

(A) 53 (37): G. Tiercy, Sur les suspensions pendulaires à lames flexibles. Ref. 2341 .

G. Tiercy, Le tremblement de terre du 25 janvier 1946 et le comportement de quelques horloges à pendule. Ref. 2342.

4 (38): G. Tiercy, Une solution approchée de mouvement pendulaire isochrone. Ref. 2343.

G. Tiercy, Récidive sismique en Valais et nouveaux faits pendulaires. Ref. 2344.

5 (39): A propos du jubilé newtonien de Londres. Note sur trois astronomes de la Suisse française des XVIIe et XVIIIe siècles. Ref 1064. 
XXVIII Übersicht über die referierten Zeitschriften

6 (40): G. Tiercy, A propos du centenaire de la découverte de Neptune. Les relations amicales et la collaboration scientifique entre Urbain Le Verrier et Emile Gautier. Ref. 1053.

7 (41): G. Tiercy, La première horloge à quartz de l'Observatoire de Genève. Ref. 2340.

8 (42): P. Challande, Remarques sur la mise au point d'une horloge à quartz. Ref. $\mathbf{2 3 1 0 .}$

R. Luthy, La réception radiotélégraphique des signaux horaires. Ref. 2320 .

Georgetown, Wash.

176. Georgetown Observatory Reprint.

Nr. 16: J.P. Hagen, Temperature gradient in the sun's atmosphere measured at radio frequencies. Ref. 6538.

\section{Glasgow}

177. Glasgow University Observatory Reprint.

Nr. 29: W. M. Smart, Adress on the award of the Gold Medal to Professor Antonie Pannekoek. Ref. 1002.

W. M. Smart, The first half of the twentieth century: a partial review. Ref. 1319.

\section{Göttingen}

178. Veröffentlichungen der Universitäts-Sternwarte zu Göttingen.

Nr. 101: P. ten Bruggencate, F. W. Jäger, Das Turmteleskop der Göttinger Sternwarte. Ref. 2108.

179. Mitteilungen der Universitäts-Sternwarte Göttingen.

Nr. 5: S. Günther, Langsame Helligkeitsänderungen bei einem Hauptreihenstern der Plejaden. Ref. 11168.

\section{Greenwich}

180. Observations made at the Royal Observatory, Greenwich, in the year 1937 in Astronomy, Magnetism and Meteorology. Ref. 2348, 6001, $6353,6660,7101,7113,7301,7401,7562,7601,7701,7801,7804$, $9119,10125,13246$.

181. Report of the Astronomer Royal to the Board of Visitors of the Royal Greenwich Observatory. Ref. 1101.

182. The Nautical Almanac and Astronomical Ephemeris for the Year 1952. Ref. 403.

183. Apparent Places of Fundamental Stars 1952. Ref. 411.

184. The Abridged Nautical Almanac for the Year 1952. Ref. 420.

185. The Air Almanac 1952. Ref. 427.

186. The Star Almanac for Land Surveyors for the Year 1952. Ref. 431.

\section{Hamburg}

187. Mitteilungen der Hamburger Sternwarte in Bergedorf.

9 74: M. Beyer, Beobachtungen von ungewöhnlichen oder wenig bekannten Veränderlichen. Ref. 11203.

75: J.v. d. Heide, Verzeichnis von 207 Sternen mit großer Eigenbewegung. Ref. 9202.

76: P. Wellmann, Die Elemente des Systems Zeta Aurigae. Ref. 11136.

77: G. Thiessen, Der Bergedorfer Zählwerkchronograph. Ref. 2246. 
78: M. Beyer, Beobachtungen von 30 zweifelhaften Veränderlichen. Ref. 11204.

79: P. Wellmann, Ein dreilinsiges Objektiv für die photographische Photometrie. Ref. 2033.

80: J. Stock, Photographische Photometrie des Sternhaufens Coma Berenices in drei Spektralbereichen mit einem photometrischen Spezialobjektiv. Ref. 12114.

81: K. Wurm, Die Temperatur des Crabnebel-Zentralsterns. Ref. 12228.

21 213: O. Heckmann, P. Jordan, W. Fricke, Zur erweiterten Gravitationstheorie. I. Ref. 4122.

214: P. Wellmann, Die Blaufärbung der Sonne am 27. September 1950. Ref. 6027.

215: W. Becker, Entfernungsbestimmung offener Sternhaufen nach der Methode der Farbdifferenzen. Ref. 12101.

216: P. Wellmann, Über das Spektrum der Nova EU Scuti 1949. Ref. 11334.

217: P. Wellmann, Nova DK Lacertae 1950. 1. Teil: Die spektrale Entwicklung und die Radialgeschwindigkeiten. Ref. 11324.

218: P. Wellmann, Veränderungen im Spektrum von FU Orionis. Ref. 11312.

219: W. Becker, Farben-Helligkeits- und Farbdifferenz-Diagramme von Plejaden und Praesepe. Ref. 12102.

220: W. Becker, Bemerkungen zum Rotationsproblem von Spiralsystemen. Ref. 12333.

221: G. Thiessen, Zur Theorie der Polarisation von $\mathbf{H}_{\alpha}$ und $\mathrm{D}_{3}$ in Protuberanzen. Ref. 6655.

188. Deutsches Hydrographisches Institut. Jahresbericht Nr. 5, 1950. Ref. 1101.

189. Nautisches Jahrbuch oder Ephemeriden und Tafeln für das Jahr 1952. Ref. 419.

\section{Hante-Provence}

190. Publications de l'Observatoire de Haute-Provence.

2 10: Tcheng Kien, Intensité de la bande 4300 A de la molécule $\mathrm{CH}$ dans les spectres des étoiles F, G, K et M. Ref. 9555.

11: Vgl. AJB 50 Ref. 12202.

12: Tcheng-Kien, Sur la classification des étoiles des classes F, G, K et M. Ref. 9556.

13: Vgl. AJB 50 Ref. 72102.

14: J.Dufay, M. Dufay, Etude du spectre d'émission du ciel nocturne, de 6800 à $9000 \AA$. Ref. 7295 .

15: J. Bigay, Photométrie globale des galaxies par la méthode de Ch. Fabry. Ref. 12407.

J. Bigay, Remarques sur la magnitude absolue moyenne et la relation vitesses radiales - magnitudes apparentes des nébuleuses extragalactiques. Ref. 12408.

J. Bigay, Photométrie globale des nébuleuses extragalactiques par la méthode de Schilt. Ref. 12409.

16: F. Lenouvel, Photomètre photoélectrique stellaire. Ref. 2231.

17: M.-T. Martel, Polarisation de la lumière diffusée de N. G. C. 7023. Ref. 12211.

18: R. Peyturaux, Sur la répartition spectrale de l'énergie au centre du disque solaire entre 6700 et 23000 A. Ref. 6416 .

19: G. Courtès, Etude de la voie lactée en lumière monochromatique $\mathrm{H} \alpha$ de 320 à $350^{\circ}$ et de 25 à $80^{\circ}$ de longitude galactique. Ref. 12309 . G. Courtès, Etude de la voie lactée en lumière monochromatique $\mathrm{H} \alpha$, de 80 à $180^{\circ}$ de longitude galactique. Ref. 12310 . 
20: Y. Andrillat, C. Fehrenbach, Le spectre de Nova Scuti 1949. Vgl. AJB 50 Ref. 11353.

21: E. M. Burbidge, G. R. Burbidge, The spectrum of HD 217050. Ref. 9514.

22: F. Lenouvel, Observations photoélectriques d'étoiles variables. Ref. 11217.

23: J. Berger, A.-M. Fringant, Etude spectrophotométrique de trois étoiles à raies métalliques. Ref. 9506.

24: M. Dufay, Une nouvelle bande de vibration-rotation de la molécule $\mathrm{OH}$ dans le spectre du ciel nocturne. Ref. 7296.

M. Dufay, La raie interdite ${ }^{4} \mathrm{~S}-{ }^{2} \mathrm{D}$ de l'atome neutre d'azote dans les spectres du ciel nocturne et du crépuscule. Ref. 7297.

25: J. Berger, A.-M. Fringant, Le spectre continu de $\lambda$ Bootis. Ref. 9508.

26: L. Divan, Sur l'absorption continue par la matière interstellaire. Ref. 12507.

27: M. Bloch, Tcheng Mao-Lin, Mesures spectrophotométriques de $\mathrm{Z}$ Andromedae. Ref. 11164.

28: Vgl. AJB 52.

29: R. Bouigue, Sur la structure et la classification des étoiles des types $\mathbf{R}$ et N. Ref. 9511.

\section{Heidelberg}

Astronomisches Rechen-Institut.

191. Astronomisch-Geodätisches Jahrbuch für 1953. Ref. 404.

192. Astronomischer Jahresbericht. 46. Band. Die Literatur der Jahre 1943 -1946. 2. Teil. Ref. 701.

193. Astronomische Grundlagen für den Kalender 1953. Ref. 412.

194. Nachrichtenblatt der Astronomischen Zentralstelle. 5. Jahrgang Nr. $1 \rightarrow 11$. Vorläufige Mitteilung Nr. 101-137.

Badische Landessternwarte.

195. Mitteilungen der Badischen Landessternwarte Heidelberg-Königstuhl.

Nr. 81: G. R. Miczaika, Über den spektroskopischen Doppelstern $\varkappa$ Draconis. Ref. 10206.

82: G. R. Miczaika, Über die spektroskopischen Doppelsterne $\eta$ Orionis und $\imath$ Pegasi. Ref. 10207.

83: G. R. Miczaika, Absolute Helligkeiten von $115 \mathrm{~B}-S t e r n e n$. Ref. 9719.

84: A. König, Bemerkungen über die Ableitung photographischer Planetenörter im Anschluß an zwei Anhaltsterne. Ref. 2405.

85: G. R. Miczaika, Die Radialgeschwindigkeitskurve von ` Orionis. Ref. 10208.

86: H. Klauder, Über den Exponenten der Temperatur im stellaren Energieerzeugungsgesetz. Ref. 5216.

\section{Helsinki}

196. Sonderdrucke des Astronomischen Observatoriums Helsinki.

G. Järnefelt, P. Kustaanheimo, An observation on finite geometries. Nicht referiert.

G. Järnefelt, Karl F. Sundman in memoriam. Ref. 1001.

G. Järnefelt, Reflections on a finite approximation to Euclidean geometry. Physical and astronomical prospects. Nicht referiert. 


\section{Helwan}

197. Royal Observatory, Helwan. Bulletin.

Nr. 40: A. H. M. Samaha, The temperature coefficients of the Helwan transit circle. Ref. 2513.

41: F. L. Whipple, S. El-Din Hamid, On the origin of the Taurid meteor streams. Ref. 8289 .

\section{Hyderabad}

198. Nizamia Observatory. Reprint.

Nr. 9: Occultations of stars by the moon observed at the Nizamiah Observatory, Hyderabad, during the year 1949. Vgl. AJB 50 Ref. 7356.

10: Occultations of stars by the moon observed at the Nizamiah Observatory, Hyderabad, during the year 1950. Ref. 7341.

\section{Istanbul}

199. Publications of the Istanbul University Observatory.

Nr. 40: E. Tü zemen, La détermination des coordonnées héliographiques des taches solaires à l'aide d'un nomogramme. Ref. 6348 .

41: W. Gleissberg, Die mittlere Sonnenfleckenkurve. Ref. 6316.

42: M. Hotinli, On the foreshortening law of sunspots. Ref. 6322 .

43: E. Tüzemen, Observations des taches solaires en 1950. Ref. 6353.

200. Istanbul Kandilli Rasathanesi.

M. Dizer, Observations des taches solaires en 1950 et 1951. (Janvier-Juin). Ref. 6353.

\section{Jena}

201. Mitteilungen der Universitäts-Sternwarte zu Jena.

Nr. 12/2: K. Güssow, Die spektrale Empfindlichkeit photographischer Schichten. Ref. 2259.

\section{Johannesburg}

202. Union Observatory. Circular.

61 (111): E. L. Johnson, W. H. van den Bos, J. A. Bruwer, Occul. tations of stars by the moon. Ref. 7341 .

E. L. Johnson, Approximate positions of minor planets, 1950. Ref. 7562.

E. L. Johnson, Photographic observations of minor planets, 1950. Ref. 7562.

W. P. Hirst, Preliminary orbits of new minor planets, 1950. Ref. 7562 .

E. L. Johnson, Photographic observations of comets, 1950. $\$ 81$. W. H. van den Bos, J.A. Bruwer, Orbits of double stars. Ref. 10128.

W. H. van den Bos, Measures of double stars - 30th series. Ref. 10125.

2 (112): W. S. Finsen, The significance of atmospheric dispersion in astronomical interferometry. Ref. 3108.

W. S. Finsen, Interferometer observations of artificial double stars. Ref. 10004.

W. S. Finsen, Interferometer measures of double stars. Ref.10125.

W. S. Finsen, Micrometer measures of double stars. Ref. 10125.

W. H. van den Bos, Orbits of double stars. Ref. 10128. 
XXXII Ủbersicht über die referierten Zeitschriften

\section{Kanzelhöhe}

203. Mitteilung aus dem Sonnenobservatorium auf der Kanzelhöhe in Kärnten.

Nr. 2: A. Bruzek, Beobachtungen über das Verhalten von Filamenten während chromosphärischer Eruptionen. Ref. 6605.

3: A. Bruzek, Aktivierung eines Filaments während einer chromosphärischen Eruption. Ref. 6603.

4: A. Bruzek, Über eine ungewöhnliche chromosphärische Eruption. Ref. 6604.

\section{Kasan}

204. Mitteilungen (Iswestija) des Astronomischen Engelhardt-Observatoriums der Lenin-Staatsuniversität Kasan.

Nr. 26: D. J. Martynow, Untersuchung des Lichtwechsels einiger veränderlicher Sterne. Ref. 11218.

A. A. Nefedjew, Die physische Libration des Mondes. Ref. 7312.

27: D. J. Martynow, Das Astronomische Engelhardt-Observatorium 1901-1951. Ref. 1217.

A. A. Nefedjew, Wassilij Pawlowitsch Engelhardt. Ref. 1038. L. D. Agafonowa, A. I. Nefedjewa, Der Meridiankreis des Astronomischen Engelhardt-Observatoriums in den Jahren 1903 -1951. Ref. 2101.

A. A. Nefedjew, Das Heliometer des Astronomischen Engelhardt-Observatoriums. Ref. 2126.

D. J. Martynow, Das Schmidt-Teleskop des Astronomischen Engelhardt-Observatoriums. Ref. 2121.

S. T. Chabibullin, Das Belkowitsch-Horizontalteleskop des Astronomischen Engelhardt-Observatoriums. Ref. 2110.

A. K. Iwanowa, Die Station zur Beobachtung der Lotschwankungen am Astronomischen Engelhardt-Observatorium. Ref. 1217.

\section{Kiel}

205. Sonderdruck der Sternwarte Kiel.

E. Vitense, Der Aufbau der Sternatmosphären. IV. Teil. Kontinuierliche Absorption und Streuung als Funktion von Druck und Temperatur. Ref. 5132.

D. Labs, Untersuchungen zur inkohärenten Streuung in Fraunhoferlinien. Ref. 5110.

H.-H. Voigt, Über die Polarisation der Strahlung in Sternatmosphären. Ref. 5134.

K. Hunger, Zur Berechnung der Intensitätsverteilung in Fraunhoferlinien. II. Teil: Berechnung der Gewichtsfunktionen bei vorgegebener Kirchhoff-Planck-Funktion mit exponentiellem Glied. Ref. 5106.

E. Vitense, Ǔber die mittleren Zustandsgrößen und spektralen Eigenschaften von Sternatmosphären in Abhängigkeit von Effektivtemperatur und Schwerebeschleunigung. Ref.5133.

D. Labs, Die Temperaturschichtung der Sonnenatmosphäre im Strahlungsgleichgewicht unter Berücksichtigung der Fraunhoferlinien. Ref. 6117.

\section{Kiew}

206. Zirkulare des Astronomischen Observatoriums der Schewtschenko-Staatsuniversität Kiew Nr. 42-54. Ref. 6353, 6660. 


\section{Kobenhavn}

207. Publikationer og mindre Meddelelser fra Kobenhavns Observatorium.

Nr. 156: M. Rudkjøbing, A photoelectric differential classification of $A$ and $F$ stars. Ref. 9539.

208. Union Astronomique Internationale. Circulaire Nr. 1298-1339.

209. Aktuelle Meddelelser fra Astronomisk Selskab Nr. 339-346.

\section{Kodaikanal}

210. Kodaikanal Observatory Bulletin.

Nr. 131: A. K. Das, Summary of prominence observations for the second half of 1949. Ref. 6660 .

211. Annual report of the Kodaikanal Observatory for the year 1949. Desgl. 1950. Ref. 1101.

\section{Krakau}

212. Rocznik Astronomiczny Obserwatorjum Krakowskiego 1951. Supplemento Internationale Nr. 22. Ref. 417, 7340, 11012.

\section{Krim}

213. Mitteilungen (Iswestija) des Astrophysikalischen Observatoriums auf der Krim.

6: G. A. Schajn, W. F. Hase, Einige Ergebnisse der Untersuchung leuchtender galaktischer Nebel. Ref. 12219.

I. S. Schklowskij, S. B. Pikelner, Die thermische Radiostrahlung der Sonne. Ref. 6553.

A. B. Gilwarg, A. B. Sewerny, Die Vervollkommnungen der Interferenzpolarisationsfilter für Sonnenbeobachtungen und ein Versuch zur kinematographischen Aufnahme von Protuberanzen. Ref. 2223.

N. A. Kosyrew, Die Theorie des inneren Aufbaues der Sterne und die Quellen der Sternenergie. Teil II. Ref. 9712.

E. S. Brodskaja, Lichtelektrische Beobachtungen von vier Uberriesen und zwei Wolf-Rayet-Sternen. Ref. 9403.

I. S. Schklowskij, Ionisation und chemische Zusammensetzung der Sonnenkorona. Ref. 6721.

A. A. Kalinjak, W. I. Krassowskij, W:B. Nikonow, Beobachtung des Gebiets um das galaktische Zentrum im Licht von etwa 10000 Á. Ref. 12313.

S. W. Nekrassowa, Bahnelemente des Bedeckungsveränderlichen V 451 Ophiuchi. Ref. 11122.

W. W. Such anowa, Spektralphotometrie zweier Protuberanzen mit einem Interferenzpolarisationsfilter. Ref. 6646.

E. R. Mustel, Ưber die Herkunft der Ausbrüche der Novae. Ref. 11305.

P. F. Schajn, Beobachtungen Kleiner Planeten und Kometen in Simeis. Ref. 7562, § 81 .

P. F. Schajn, Die Entdeckung des neuen periodischen Kometen 1949 e. Ref. 8170.

S. B. Pikelner, W. W. Suchanowa, K. K. Tschuwajew, E. F. Schaposchnikowa, Spektrohelioskopische Beobachtungen 1949. Ref. 6660.

7: E. R. Mustel, Das Balmerdekrement im Spektrum der Flocculi. Ref. 6837.

I. S. Schklowskij, Die Natur des infraroten Nachthimmellichts. Ref. 22113. 
A. B. Sewerny, Versuch einer Spektralphotometrie Fraunhoferscher Linien mittels Spektrographen und Fabry-Etalon. Ref. 6423. G. A. Schajn, Die Zentralintensitäten der starken Absorptionslinien in Sternspektren. Ref. 9541.

G. A. Schajn, W. F. Ha se, Über eine besondere Klasse diffuser gasförmiger Nebel. Ref. 12220.

W. F. Hase, G. A. Schajn, Zweites Verzeichnis diffuser Nebel. Ref. 12209.

S. B. Pikelner, Induktionserscheinungen in der Sonnenatmosphāre. Ref. 6341.

G. A. Monin, A. B. Sewerny, Der Spektroheliograph des Astrophysikalischen Observatoriums auf der Krim. Ref. 1220.

E. R. Mustel, Die Energieverteilung im kontinuierlichen Spektrum der Novae. Ref. 11306.

G. A. Schajn, Radialgeschwindigkeiten von 73 Sternen. Ref.9216. I. I. Nasarowa, Spektralphotometrie der $\mathrm{Ca}$ - und Cat-Linien in einer chromosphärischen Eruption. Ref. 6629.

E. S. Brodskaja, Neuer spektroskopisch entdeckter T TauriVeränderlicher. Ref. 11242.

P. F. Schajn, Beobachtungen Kleiner Planeten in Simeis 1950. Ref. 7562.

W. A. Albizkij, Beobachtungen Kleiner Planeten. Ref. 7562.

\section{Kyoto}

214. Contributions from the Institute of Astrophysics, Kyoto University.

Nr. 10: S. Miyamoto, On the ultraviolet emission of the chromosphere. Ref. 6622 .

11: S. Miyamoto, On the excitation of helium in the chromosphere. Ref. 6623.

12: T. Ishizu, Note on some resonance lines in the ultraviolet spectrum of the sun. Ref. 6407.

13: S. Miyamoto, Radiation field of extended stellar atmospheres. Ref. 5113.

14: S. Miyamoto, On the anomalous excitation of the chromospheric hydrogen spectrum. Ref. 6624.

15: J. Jugaku, On the radiation field of the extended stellar atmospheres. Ref. 5107.

16: S. Miyamoto, The solar noise from the coronal prominences. Ref. 6546.

17: S. Miyamoto, Kinetic temperature of the chromosphere. Ref. 6625 .

215. Kwasan Observatory Reprints.

Nr. 1-2: T. Mitani, Photographic observations of comets.Vgl. AJB 50 § 81. T. Mitani, Visual observations of comets made with the $30 \mathrm{~cm}$ refractor. Vgl. AJB 50 § 81 .

3: J. Ueta, The effect of the plumb line deviation in longitude on the observation of occultations and transits by the moon. Vgl. AJB 50 Ref. 3212 .

\section{La Plata}

216. Publicationes del Observatorio Astronomico de la Universidad Nacional de La Plata. Serie Astronomica.

20 3: J. Landi-Dessy, La binaria p Eridani. Ref. 10111.

27 2: P. Sconzo, Calculo de una orbita a partir de una solucion approximada. Ref. 4517. 
217. Publicaciones del Observatorio Astronomico de la Universidad Nacional de La Plata. Serie Circular.

Nr. 8 : Comunicaciones del Departamento de Astrofisica:

L. Gratton, J. Dvinianin, C. Jaschek, La velocidad del sol con respecto a la velocidad circular en la galaxia. Ref. 12339. L. Gratton, M. I. Corvalán, Sobre el calculo de la abundencia hidrogeno-helio en las binarias. Ref. 10005.

N. Tapia, Nova Scorpi 1950. Ref. 11332.

L. Gratton, El maximo secundario de T CrB. Ref. 11319.

9 : M. Itzigsohn, P. Sconzo, Observaciones y calculos de planetitas. Ref. 7517, 7531.

10 : Primer Congreso Interobservatorios Nacionales 23, 24 y 25 Noviembre de 1950. Ref. 1180.

1: S. Gershánik, Mejoras en el planeto de la relación entre el signo de $\mathbf{P}$ y las fuerzas generadoras de un terremoto. Nicht referiert.

2: J. L. Rayces, Aplicación del método de Foucault al control de espejos paraboloidales astronómicos. Ref. 2025.

3: P. Sconzo, Téoria aproximada del planetita (671) Carnegia. Ref. 7532.

4: J. Bobone, El asteroide Icarus (su órbita e importancia astronómica). Ref. 7503.

5: M. Dartayet, J. L. Dessy, Búsqueda de estrellas variables en las nubes de Magallanes. Ref. 12411.

6: A. Wilkens, El problema de la multiplicidad de las órbitas cometarias parabólicas. Ref. 4520.

7: A. A. Baldini, Nuevos adelantos en Geodesia. Nicht referiert.

8: A. A. Baldini, Recepción de señales radio-horarias rítmicas, por el método de coincidencias. Ref. 2305.

9: H. Wilkens, Recuentos estelares en algunas regiones galácticas Ref. 12533.

10: J. Sahade, J. Landi-Dessy, La binaria espectroscópica Boss 4496. Ref. 10212.

11: J. Sahade, J. Landi-Dessy, El espectro de RR Telescopii en 1949. § 112 .

12: L. Gratton, Contribución a la teoría de las atmósferas estelares. Ref. 5105.

13: R.P. Platzeck, Deformaciones del espejo principal del reflector de Bosque Alegre. Ref. 2510.

14: S. J. Sla ucitajs, Sobre la determinación de las correcciones de trazo de los Círculos Graduados y errores de graduación del Circulo I del Anteojo Meridiano Repsold del Observatorio Astronómico de La Plata. Ref. 2515.

15: L. Slaucitajs, Investigaciones sobre la variación secular geomagnética. Nicht referiert.

16: A. A. Baldini, Determinación de tiempo y latitud. Ref. 3102.

17: A. Corpaciu, El Túnel Transandino. Nicht referiert.

18: L. Gratton, Mediciones de espectros de estrellas $K$. en gran dispersión. Ref. 9520.

19: J. Sahade, Las variables de eclipses S Velorum y R Arae. § 112.

20: A. Wilkens, Temperaturas espectrográficas estelares. Ref. 9606 .

21: P. Sconzo, Planteo y resolución práctica del problema de mejoramiento de una órbita elíptica, aprovechando todas las observaciones de una oposición. Ref. 4516.

22: M. Itzigsohn, Dos aspectos del trabajo en Astronomía Extrameridiana. Ref. 3005.

23: S. Gershánik, P. J. Sierra, El sismógrafo Sprengnether del Observatorio de La Plata. Nicht referiert. 
XXXVI Uthersicht über die referierten Zeitschriften

24: N. Tapia, Resultados de las determinaciones de las magnitudes fotográficas de las estrellas de la C. P. D. entre 72 y 82 grados de declinación austral, hasta magnitud novena. Ref. 9423.

25: L. Gratton, C. J. Lavagnino, Medición de velocidades radiales de AI Velorum. \$112.

26: R. P. Cesco, Nueva expresión de las perturbaciones absolutas. Contribución al problema de órbitas que se cruzan. Ref. 4202.

27: A. Wilkens, Observaciones en el cielo austral para el progreso de la dinámica estelar. Ref. 1255.

28: L. Gratton, Necesidades observacionales astrofísicas en el hemisferio austral. Ref. 9002.

218. Publicaciones del Observatorio Astronomico de la Universidad Nacional de La Plata. Serie Especial.

Nr. 11: De Ceres a Evita. Un siglo y medio de desenbrimientos de planetitas.

Primera Parte: El planetita Evita. Ref. 7540.

Segunda Parte: P. Sconzo, Conmemoracion historica del descubrimiento del primer asteroide "Ceres». Ref. 1453.

12: G. O. Wallbrecher, Memoria anual correspondiente al año 1949. Ref. 1101.

13: L. Slaucitajs, La observación del campo geomagnetico. Nicht referiert.

14: P. Sconzo, Nuevas tablas para el cálculo de las efemérides de los asteroides. Ref. 4523.

15: G. O. Wallbrecher, Memoria anual correspondiente al año 1950. Ref. 1101.

\section{Leiden}

219. Annalen van de Sterrewacht te Leiden.

20 8: C. J. van Houten, First ephemerides of thirty variable stars in or near the constellation Vela. Ref. 11212.

9: A. D. Fokker, Investigation of nineteen variables in a region in or near the constellation Sagittarius and of one variable in the constellation Vela. Ref. 11209.

\section{Lembang}

220. Annals of the Bosscha Observatory Lembang (Java).

9 1: G. V. Simonow, Visual measures of double stars. 6 $6^{\text {th }}$ series 1936-1940. Ref. 10125.

221. Lembaga Astronomi. Bosscha Sterrenwacht Lembang, Java. Report for the years 1949-1950. Ref. 1101.

\section{Leningrad}

222. Bulletin des Instituts für Theoretische Astronomie der Akademie der Wissenschaften der UdSSR.

49 (62): W. W. Sack, D. K. Kulikow, Bestimmung der definitiven Bahn des Kometen 1925 VII (Schajn-Comas Sola). Ref. 8133.

D. K. Kulikow, Formeln und Tabellen zur differentiellen Verbesserung parabolischer Bahnen. Ref. 4521.

F. B. Chanina, Die Bahn des Planeten (534) Nassovia. Ref. 7510. F. B. Chanina, Bahnverbesserung des Planeten (1340) Yvette. Ref. 7511 .

A. I. Boshkowa, Genäherte Verbesserung der Bahnelemente des Planeten (1078) Mentha. Ref. 7505. 
10 (63): G. A. Tschebotarjow, Anwendung der periodischen Bahnen auf die Untersuchung der Bewegung Kleiner Planeten. Ref. 7537.

5 (64): D. W. Sagrebin, Ửber die Berücksichtigung des empirischen Gliedes bei der Aufstellung einer Mondephemeride. Ref. 7313.

G. A. Tschebotarjow, L. J. Piuss, E. N. Lemechowa, Die absolute periodische Bahn des Hestia-Typs. Ref. 7538.

N. F. Bojewa, Der Komet 1941e Du Toit-Neujmin-Delporte. Ref. 8142 .

W. F. Proskurin, T. I. Maschinskaja, Heliozentrische Bahnkoordinaten von Jupiter. Ref. 7608.

223. Astronomisches Jahrbuch der UdSSR für das Jahr 1954. Ref. 405.

224. Ephemeriden Kleiner Planeten für das Jahr 1952. Ref. 7501.

\section{Liège}

225. Institut d'Astrophysique. Collection in $4^{\circ}$ de Spectroscopie et Astrophysique.

Nr. 28: P. Swings, Spectroscopic problems of astronomical interest. Ref. 259.

29: E. Etienne, P. le Goff, B. Rosen, Etude au laboratoire des conditions d'excitation des bandes d'émission "cométaires". Ref. 8006.

30: M. Migeotte, L. Neven, Nouvelles raies de méthane tellurique. Ref. 7235.

31: P. Ledoux, Sur la stabilité gravitationnelle d'une nébuleuse isotherme. Ref. 5305 .

226. Institut d'Astrophysique. Collection de Mémoires in $8^{\circ}$.

Nr. 330: E. Lebon, Note sur la température de la comète de Encke. Ref. 8118.

331: E. Sauvenier-Goffin, Note sur les pulsations non-radiales d'une sphère homogène compressible. Ref. 5229.

332: D. Jehoulet, Note sur l'asymétrie des courbes de lumière et de vitesses radiales des céphéides. Ref. 11150.

333: J.W. Swensson, Prédictions de raies atomiques dans l'infrarouge. Ref. 258.

334: P. Ledoux, The nonradial oscillations of gaseous stars and the problem of Beta Canis Majoris. Ref. 5220.

335: M. Migeotte, L. Neven, L'activité de la mission scientifique belge à la station du Jungfraujoch en 1950 et 1951 . Ref. 1216 .

336: B. Rosen, I. Ottelet, Utilisation de la cathode creuse et d'un four à vide en spectroanalyses. Ref. 2240 .

337: P. Swings, La physico-chimie des comètes. Ref. 8024.

\section{London}

227. Communications from the University of London Observatory.

Nr. 6: E. M. Burbidge, G. R. Burbidge, Hydrogen and helium line intensities in some Be stars. Ref. 9513.

7: Stellar parallaxes determined at the University of London $\mathrm{Ob}$ servatory, Mill Hill (second list). Vgl. AJB 50 Ref. 9306.

\section{Los Angeles}

228. University of California, Los Angeles. Astronomical Papers.

Nr. 12: S. Herrick, Step-by-step integration of $\ddot{\mathrm{x}}=f(x, y, z, t)$ without a "corrector". Ref. 105. 
XXXVIII Übersicht über die referierten Zeitschriften

Lund

229. Annals of the Observatory of Lund.

Nr. 11: A. Reiz, Meridian observations of faint AG stars. Observations made with the Repsold meridian circle of the Lund Observatory during the years $1943-45$ and reduced to the equinox of 1950 . Ref. 9107.

230. Meddelande från Lunds Astronomiska Observatorium.

(1) Nr. 176: A. Reiz, On a special case of a quadrature formula of Christoffel. Ref. 112.

177: N. Hansson, An attempt to a photographic calibration of a tungsten ribbon lamp. Ref. 225.

178: F. Nettelblad, Recordings of scintillation. Ref. 72152.

\section{Lwow (Lemberg)}

231. Zirkular des Astronomischen Observatoriums der Franko-Staatsuniversität Lwow Nr. 4-17, 1947-1950. §63, 66, 73, 75, 81, 82, 112, 113.

\section{Lyon}

232. Publications de l'Observatoire de Lyon.

(1) 5 15: Tcheng-Kien, Recherches sur les bandes de CH dans les spectres stellaires et solaire. Ref. 9554 .

16: J. Dufay, Bandes d'émission des molécules $\mathrm{OH}$ et $\mathrm{O}_{2}$ dans le spectre du ciel nocturne, entre 9000 et 11000 A. Ref. 7294.

17: Tcheng Mao-Lin, Mesures de la quantité d'ozone contenue dans l'atmosphère par spectrophotométrie photographique du ciel bleu au zénith. Ref. 7248.

18: Tcheng Mao-Lin, Etude spectrophotométrique de la polarisation de la lumière du ciel bleu au zénith de 3050 à 4500 A. Ref. 7249 .

19: J. H. Bigay, Introduction à la photométrie des nébuleuses extragalactiques. Ref. 12410.

20: J. H. Bigay, Photométrie photographique des nébuleuses extragalactiques. Ref. 12405.

233. Publications de l'Observatoire de Lyon.

(2) 1 7: J. P. Chassany, Résumé de 70 années d'observations météorologiques à Saint-Genis-Laval. Nicht referiert.

8: Cent années d'observations de la température à Lyon. Nicht referiert.

\section{Madrid}

234. Boletin Astronomico del Observatorio de Madrid.

4 4: E. Gullón, Protuberancias solares. Resumen de las observaciones realizadas durante el año 1950 . Ref. 6660.

E. Gullón, Altura de la cromosfera en 1950. Ref. 6660 .

E. Gullón, Observaciones de manchas solares (Numeros relativos de Wolf). Observaciones realizadas en los observatorios de Madrid, Valencia, Cartuja (Granada) y Ebro en 1950. Ref. 6353.

R. Carrasco, Observaciones fotográficas de pequeños planetas (Posiciones exactas). Ref. 7562.

235. Universidad de Madrid - Facultad de Ciencias. Seminario de Astronimía y Geodesia. Publicación.

Nr.6: R. Carrasco, J. M. Torroja, Rectificación de la órbita del asteroide 1371 «Resi». Ref. 7562. 
7: J.M. Torroja, R. Carrasco, Rectificación de la órbita del asteroide 1560 (1942 XB) y efemérides para la oposición de 1951. Ref. 7562.

8: L. Siegrist, Orbita provisional del sistema visual 728-32 Orionis. Ref. 10128.

9: Efemérides de 79 asteroides para la oposición de 1952. Ref. 7562.

10: J. Pensado, Orbita provisional de $\Sigma 1883$. Ref. 10128.

236. Anuario del Obervatorio Astronómico de Madrid para 1952. Ref. 439, 1224.

\section{Manchester}

237. Astronomical Contributions from the University of Manchester. Series II. Jodrell Bank Reprints.

(2) 1 42: A. C. B. Lovell, The summer daytime meteor streams. Ref. 8273.

43: A. C. B. Lovell, Radio astronomy. Ref. 12614.

44: A. C. B. Lovell, The new science of radio astronomy. Ref. 12615.

45: A. Aspinall, G. S. Hawkins, The summer daytime meteor streams of 1949 and 1950 . I. Measurement of the radiant positions and activity. Ref. 8268.

46: J. G. Davies, J.S. Greenhow, The summer daytime meteor streams of 1949 and 1950. II. Measurement of the velocities. Ref. 8272.

47: M. Almond, The summer daytime meteor streams of 1949 and 1950. III. Computation of the orbits. Ref. 8267.

48: A. Aspinall, J. A. Clegg, G. S. Hawkins, A radio echo apparatus for the delineation of meteor radiants. Ref. 2104.

49: A. Maxwell, Radio emission from the sunspot of central meridian passage 1950 June 14. Ref. 6556.

50: C. G. Little, A. Maxwell, Fluctuations in the intensity of radio waves from galactic sources. Ref. 12613 .

51 : C. G. Little, A diffraction theory of the scintillation of stars on optical and radio wave-lengths. Ref. 72149.

52: M. Almond, J. G. Davies, A. C. B. Lovell, The velocity distribution of sporadic meteors. I. Ref. 8202 .

53: R. H. Brown, C. Hazard, Radio emission from the Andromeda nebula. Ref. 12604.

54: J.A. Clegg, R. L. Closs, Plasma oscillations in meteor trails. Ref. 8213.

55: R. H. Brown, C. Hazard, A radio survey of the Cygnus region. I. The localized source Cygnus (1). Ref. 12605.

56: Nicht eingegangen.

57-61: Vgl. AJB 52.

62: A.C. B. Lovell, J. G. Davies, Radar tracks shooting stars. Ref. 2119.

63: Vgl. AJB 52.

64: A. C. B. Lovell, Jodrell Bank Experimental Station, University of Manchester. Ref. 1101.

238. Astronomical Contributions from the University of Manchester. Series III.

(3) Nr. 3: W. H. Ramsey, On the constitutions of the major planets. Ref. 6227.

\section{Milano-Merate}

239. Contributi dell'Osservatorio Astronomico di Milano-Merate. Nuova Serie.

Nr. 31: A. Kranjc, Misure fotografiche di stelle doppie. Ref. 10125.

32: L. Volta, Emilio Bianchi. Ref. 1001. 
33: F.Zagar, Questioni dinamiche riguardanti gli ammassi stellari sferici. Ref. 12130.

34: C. Lombardi, Perturbazioni generali approssimate di (1423) Jose. Ref. 7562.

35: A. Masani, Considerazioni su qualche aspetto del problema delle Cefeidi. Ref. 11154.

36: A. Masani, Il nuovo fotometro elettronico stellare dell'Osservatorio di Merate. Ref. 2232.

240. Osservatorio astronomico di Brera in Milano. Articoli generali del calendario. Anno 1952. Ref. 440.

\section{Mizusawa}

241. Publications of the International Latitude Observatory of Mizusawa.

1 1: T. Hattori, Latitude observations with floating zenith telescope at Mizusawa. Part 1. Ref. 13217.

\section{Moskau}

242. Publikationen (Trudy) des Staatlichen Astronomischen Sternberg-Instituts.

17 1: Astronomie an der Universität Moskau. Ref. 1227.

20 : I. S. Schklowskij, Die Ionisation der Chromosphäre und Protuberanzen und das Problem der Dichteverteilung in der Chromosphäre. Ref. 6642.

P. P. Parenago, Untersuchung der Raumgeschwindigkeiten der Sterne. Ref. 12344.

P. P. Parenago, A. G. Masse witsch, Untersuchung der MasseRadius-Leuchtkraft-Beziehung. Erster Teil. Bestimmung der empirischen Masse-Radius-Leuchtkraft-Beziehung. Ref. 9725.

A. G. Massewitsch, P. P. Parenago, Untersuchung der MasseRadius-Leuchtkraft-Beziehung. Zweiter Teil. Theoretische Deutung der empirischen Beziehungen. Ref. 9717.

N. Moissejew, Über einige vereinfachte, mit Hilfe der Übermittelung des eingeschränkten elliptischen Dreikörperproblems erhaltene Grundschemen der Himmelsmechanik. 1. Über die gemittelten Varianten des eingeschränkten elliptischen ebenen Dreikörperproblems. Ref. 4217.

243. Mitteilungen (Soobschtschenija) des Staatlichen Astronomischen SternbergInstituts.

Nr. 56 : M. I. Uranowa, Eigenbewegungen von 93 Sternen. Ref. 9207. M. I. Uranowa, Utber Sterne mit großen Eigenbewegungen in der Umgebung der Nova Persei 1901. Ref. 9208.

A. M. Losinskij, Eigenbewegungen von neun veränderlichen Sternen. Ref. 9203.

T. A. Uranowa, Über die Bewegung von UZ Aurigae. Ref. 11229. R. M. Dsigwaschwili, D. S. Chawtassi, Ưber die Raumgeschwindigkeit von RR Geminorum. Ref. 11146.

60*: S. M. Polosk ow, Die Dichte der Materie in den Kometenköpfen und -schweifen, die Masse des gasförmigen Anteils in den Kometen und die Masse der Kometen. Ref. 8020.

W. Berditschewskaja, Ưber den Ionisationszustand der bewegten Gase in den Sternatmosphären. Ref. 5101.

G. Iwanow-Cholodny, Utber den Aufbau und die chemische Zusammensetzung der roten Zwerge. Ref. 5214.

70*: W. W. Podobed, Untersuchung des Meßapparats für astronomische Aufnahmen. Ref. 2512.

73 : P. G. Kulikowskij, Pawel Karlowitsch Sternberg. Ref. 1029. 
244. Zirkular des Staatlichen Astronomischen Sternberg-Instituts Nr. 49-55. Zeitdienst 1950 Oktober bis 1951 April.

245. Staatliches Astronomisches Sternberg-Institut und Lomonossow-Staatsuniversität Moskau.

M. S. Swerew, Katalog schwacher Sterne. Verzeichnis der Sterne des Fundamentalkatalogs von 931 Sternen, von denen 645 Deklinationen nördlich von $-30^{\circ}$ besitzen. Ref. 9112 .

M. S. Swerew, Katalog schwacher Sterne. Verzeichnis von 5120 Sternen mit Deklinationen von $+90^{\circ}$ bis $+30^{\circ}$. (Erster Teil des Katalogs schwacher Sterne). Ref. 9110.

M. S. Swerew, Katalog schwacher Sterne. Verzeichnis von 10235 Sternen mit Deklinationen von $+30^{\circ}$ bis $-30^{\circ}$. (Zweiter Teil des Katalogs schwacher Sterne.) Ref. 9111.

246. Akademie der Wissenschaften der UdSSR und Staatliches Astronomisches Sternberg-Institut.

B. W. Kukarkin, P. P. Parenago, Dritter Nachtrag zur ersten Ausgabe des Generalkatalogs Veränderlicher Sterne. Ref. 11004.

\section{Mount Hamilton}

247. Contributions from the Lick Observatory.

(2) Nr. 32: O. J. Eggen, Photoelectric studies. V. Magnitudes and colors of classical Cepheid variable stars. Ref. 11148.

33: H. F. Weaver, The identification of d'Agelet's Nova Sagittae of 1783. Ref. 11329

34: G. E. Kron, J. L. Smith, Red and infrared magnitudes for 125 stars in ten areas. Ref. 9418.

35: O. J. Eggen, Photoelectric studies. VI. Color-luminosity arrays for stars in Praesepe and M 39. Ref. 12107.

36: O. J. Eggen, Photoelectric studies. VII. Color and magnitude systems for brighter stars and the color-spectral-type relation. Ref. 9410.

37: O. J. Eggen, Photoelectric studies. VIII. Positional effect in photomultipliers and some revised magnitudes in the North Polar Sequence and Harvard region C 12. Ref. 9411.

248. Lick Observatory Bulletin.

Nr. 524: H. M. Jeffers, Photographic measures of double stars. Ref. 10125.

525: H. M. Jeffers, S. Vasilevskis, Observations of comets and asteroids. $\$ 81$, Ref. 7562.

\section{Mount Stromlo}

Siehe unter Canberra.

\section{Hount Wilson and Mount Palomar}

249. Mount Wilson and Palomar Observatories. Reprint.

Nr. 30: P. W. Merrill, Displaced calcium lines in the spectrum of HD 190073 . Ref. 9533.

31: R. F. Sanford, The spectra and orbits of AR Lacertae. Ref. 11131.

32-33: Vgl. AJB 50.

34: J.L. Greenstein, R. S. Richardson, Lithium and the internal circulation of the sun. Ref. 6106.

35: A. R. Sandage, A. J. Hill, The solar curve of growth for lines of Cr I. Ref. 6422 . 
36: P. W. Merrill, The spectrum of $\mathrm{BD}+11^{\circ} 4673$ during the years 1942-1950. Ref. 11179.

37: Vgl. AJB 50.

38: H. W. Babcock, The magnetically variable star HD 125248. Ref. 9803.

39: J. L. Greenstein, A search for $\mathrm{He}^{3}$ in the sun. Ref. 6114 .

40: L. Davis jr., J.L. Greenstein, The polarization of starlight by aligned dust grains. Ref. 12537.

41: W. C. Miller, P.W. Merrill, Spectroscopic observations of Be stars. Ref. 9535.

42: F. Zwicky, Tests for the cosmological time scale. Ref. 4162.

E. Pettit, The eruptive prominence of August 7, 1950. Ref. 6660.

43: J. L. Greenstein, T. Page, Negative hydrogen ions in planetary nebulae. Ref. 12205.

44: P. W. Merrill, The spectrum of XX Ophiuchi in 1949 and 1950. Ref. 11180.

45: R. F. Sanford, Radial velocities of FF Aquilae. Ref. 11157.

46: R.F. Sanford, Coudé radial velocities of TU Cassiopeiae, DT Cygni, and U Vulpeculae. Ref. 11156.

47: F. Zwicky, The Coma cluster of galaxies. Ref. 12445. J. M. Parker, Sunspot activity during 1950. Ref. 6353.

H. W. Babcock, S. Burd, The strong magnetic field of HD 133029. Ref. 9804.

E. Pettit, The eruptive prominence of December 23, 1950. Ref. 6660 .

E. Pettit, The eruptive prominence of January 17, 1951. Ref. 6660 .

Summary of Mount Wilson magnetic observations of sunspots for November and December, 1950. Ref. 6353.

48: P. W. Merrill, Displaced helium lines in the spectrum of BD $+11^{\circ} 4673$. Ref. 11181.

49: E. R. Herzog, F. Zwicky, The Palomar search for novae and the light-curves of the three novae of 1950 in Scorpius. Ref.11331, $11332,11333$.

D. B. McLaughlin, The spectra of three novae in Scorpius. Ref. 11331, $11332,11333$.

Summary of Mount Wilson magnetic observations of sunspots for January and February, 1951. Ref. 6353.

50: R. S. Richardson, Characteristics of solar flares. Ref. 6636.

51: H.D. Babcock, H.W. Babcock, The ruling of diffraction gratings at the Mount Wilson Observatory, Ref. 2203.

52: O. C. Wilson, L. H. Aller, The structure of the planetary nebula IC 418. Ref. 12226.

53: I. S. Bowen, The Palomar Observatory. Ref. 1230.

54: O. C. Wilson, A. Abt, The Of-type spectroscopic binary $\mathrm{BD}+40^{\circ} 4220$. Ref. 10211 .

55: E. P. Hubble, Explorations in space. The cosmological program for the Palomar telescopes. Ref. 12418.

56: Los Angeles meeting of the Astronomical Society of the Pacific. Ref. 1104.

E. Pettit, The motions of eruptive prominences. Ref. 6634 .

P. W. Merrill, I. S. Bowen, The spectrum of RS Ophiuchi in May 1951. Ref. 11325.

Summary of Mount Wilson magnetic observations of sunspots for May and June, 1951. Ref. 6353.

Summary of Mount Wilson magnetic observations of sunspots for March and April, 1951. Ref. 6353. 
57 : Stellar radial-velocity programs of Pacific Coast Observatories. Ref. 9211, 9213, 9215.

R. E. Wilson, A new general catalogue of radial velocities. Ref. 9217.

250. Mount Wilson and Palomar Observatories. Annual report of the director. Ref. 1101.

251. Catalogue of Photographs and Slides from the Mount Wilson and Palomar Observatories. Ref. 1230.

\section{München}

252. Veröffentlichungen der Sternwarte München.

3 17: P. Labitzke, Rektaszensionen von 302 Fundamentalsternen, beobachtet am gebrochenen Passageninstrument. Ref. 9104.

18: F. Schmeidler, Absolute Deklinationen von 141 Sternen des GC und das Deklinationssystem des Münchener Vertikalkreises. Ref. 9108.

19: W.Strohmeier, Ein Doppelfernrohr für visuelle Photometrie. Ref. 2137.

20: F. Schmeidler, Turbulenz bei thermisch stabiler Schichtung im Sterninnern. Ref. 5233.

4 1: R. Kühn, Der Licht- und Farbwechsel einiger RR Lyrae-Sterne. Ref. 11151.

2: H. Bucerius, Zu Dirichlets Ableitung des Ellipsoidpotentials. Ref. 18105.

3: M. Sauer, Verbesserte Methode zur photographischen Registrierung von Sterndurchgängen. Ref. 2410.

4: H. Bucerius, Bahnbestimmung als Randwertproblem. III. Ref. 4503.

5: F. Schmeidler, Messungen von $\zeta$ Cancri C. Ref. 10118.

253. Mitteilungen der Sternwarte München.

1 4: H. Bucerius, Über die Entstehung des Saturnringes. Ref. 7706.

\section{Münster}

254. Mitteilungen der Universitäts-Sternwarte Münster.

Nr. 1: O. Günther, Photometrische Beobachtungen von 4 Kleinen Planeten. Ref. 7556.

\section{Napoli (Capodimonte)}

255. Osservatorio Astronomico di Capodimonte-Napoli. Contributi Astronomici Serie II.

3 15: A. Colacevich, Su una possibile deviazione della luce attraverso la nebulosa delle Pleiadi. Ref. 12106.

4 9: A. Kranje, Sull'integrazione della equazione di Ritter Emden. Ref. 106.

10: A. Kranjc, Atmosfere intermedie fra binarie strette. Ref. 10007.

256. Osservatorio Astronomico di Capodimonte-Napoli. Collezione Miscellanea.

1 1: A. Colacevich, Un problema di ottica strumentale in Astronomia. Ref. 2006.

257. Osservatorio Astronomico di Capodimonte-Napoli. Contributi geofisici Serie II.

Nr. 11: E. Guerrieri, Anomalie climatologiche osservate a Napoli tra il 1948 ed il 1950. Nicht referiert. 
XLIV Übersicht über die referierten Zeitschriften

\section{Neuchâtel}

258. Rapport du directeur de l'Observatoire Cantonal de Neuchâtel. Ref. 1101.

\section{New Haven}

259. Transactions of the Astronomical Observatory of Yale University.

23: I. Barney, Supplementary Volume to the Yale Zone Catalogues, $-30^{\circ}$ to $+30^{\circ}$. Ref. 9102 .

Oslo

260. Institutt for Teoretisk Astrofysikk Blindern-Oslo. Småtrykk.

Nr. 1: J. Wasiutyński, Solsystemets opprinnelse. Ref. 5534.

\section{Ottawa}

261. Contributions from the Dominion Observatory Ottawa.

2 10: W. S. McClenahan, E. G. Woolsey, R. W. Tanner, Photographic registration of circle settings with the Ottawa meridian circle telescope. Ref. 2122.

\section{0xiord}

262. Communications from the University Observatory, Oxford.

Nr. 31: J. B. Sykes, Approximate integration of the equation of transfer. Ref. 5126.

\section{Padova}

263. Osservatorio Astronomico di Padova. Pubblicazioni e Ristampe.

Nr. 96: G. Mannino, Ricerca sul moto proprio della stella $\mathrm{BD}+36^{\circ} 2147$ $=$ Lalande 21185. Ref. 9205 .

264. Osservatorio astronomico di Padova. Elementi astronomici per l'anno bisestile 1952. Ref. 443.

\section{Palermo}

265. Osservatorio di Palermo. Elementi astronomici per l'anno 1951. Ref. 444.

\section{Paris}

266. Contributions de l'Institut d'Astrophysique de Paris. Série A.

(A) Nr. 78: P. Couteau, Sur une détermination de la température effective d'une naine blanche montrant des raies de l'hydrogène en légère émission. Ref. 9601.

79: G. de Va ucouleurs, Observations des discontinuités crépusculaires. Ref. 72160 .

80: V. Kourganoff, Exact source functions by an extension of Chandrasekhar's limiting process. Ref. 5108.

81: P. van de Kamp, Long-focus photographic astrometry. Ref. 10006.

82: E. Schatzman, Sur les particules diffusantes dans l'atmosphère de Mars. Ref. 7413.

83: $R$. Peyturaux, Sur la répartition spectrale de l'énergie au centre du disque solaire entre 6700 et $23000 \AA$. Ref. 6416 .

84: C. Pecker, Sur l'équilibre de la zone convective des atmosphères des étoiles. Ref. 5114. 
85: C. Pecker, Sur le raccord d'une zone adiabatique et d'une zone radiative dans l'atmosphère des étoiles. Ref. 5115.

86: R. Peyturaux, Sur la température de brillance du centre du disque solaire entre 6700 et $23000 \mathrm{~A}$ déduite des observations récentes. Ref. 6417.

87: C. Pecker, Sur les atmosphères possédant une zone convective en équilibre adiabatique. Ref. 5116.

88: P. Dumézil-Curien, Entropie d'un mélange de gaz en équilibre d'excitation et d'ionisation. Ref. 212.

89: C. de Jager, J.-C. Pecker, Interprétation des mesures de vitesses radiales dans les granules solaires. Ref. 6408.

J.-C. Pecker, C. de Jager, Largeurs équivalentes des raies solaires et microturbulence, Ref. 6415.

90: E. Schatzman, L'isotope ${ }^{3} \mathrm{He}$ dans les étoiles. Application à la théorie des novae et des naines blanches. Ref. 5232.

91: J. Berger, R. Canavaggia, D. Chalonge, A.-M. Fringant, Etude spectrophotométrique de quelques sous-naines. Ref. 9505.

92: J. Berger, A.-M. Fringant, Etude spectrophotométrique de trois étoiles à raies métalliques. Ref. 9506.

93: J. Berger, R. Canavaggia, D. Chalonge, A.-M. Fringant, La discontinuité de Balmer de quelques étoiles à atmosphère étendue. Ref. 9507.

94: R. Peyturaux, Sur l'opacité de la photosphère solaire entre 6700 et 23000 A. Ref. 6418.

95: J. Berger, A.-M. Fringant, Le spectre continu de $\lambda$ Bootis. Ref. 9508.

96: L. Divan, Sur l'absorption continue par la matière interstellaire. Ref. 12507.

97: D. Chalonge, Dix ans d'observations astronomiques au Jungfraujoch. Ref. 1216.

98: R. Peyturaux, Sur un modèle d'atmosphère solaire déduit des mesures sur le fond continu du spectre. Ref. 6419.

99: H. Mineur, Tentatives de calcul numérique des intégrales doubles. Ref. 110.

100: Vgl. AJB 52.

101: Vgl. AJB 52.

102: H. Mineur, Recherche d'une influence possible de la lune sur les précipitations atmosphériques. Ref. 7311.

103: P. Dumézil-Curien, Sur la relation de phase luminosité-vitesse des céphéides. Ref. 11147.

267. Contributions de l'Institut d'Astrophysique de Paris. Série B.

(B) Nr. 61: R. Cayrel, Contribution à l'étude de l'équilibre radiatif (Influence de la zone convective). Ref. 5103 .

62: P. Dumézil-Curien, Sur certaines réactions nucléaires d'importance astrophysique. II. Création de paires d'électrons. Influence sur la formation des neutrons. Ref. 213.

63: P. Dumézil-Curien, E. Schatzman, Sur certaines réactions nucléaires d'importance astrophysique. III. Hydrogène brusquement porté à une température de plusieurs milliards de degrés. Ref. 214.

64: R. Michard, Remarques sur l'effet Evershed. Ref. 6332.

65: J.-C. Pecker, Contribution à la théorie du type spectrale. I-V. Vgl. AJB 50 Ref. 5117, 5128, 5129, 5130, 6120.

66: P. Couteau, Sur une relation entre la position des naines blanches dans le diagramme de Russell, leur concentration en hydrogène et leur masse. Ref. 9702. 
67: R. Rigollet, Sur une étude du radiant des Bootides par la méthode télescopique et sur un essai de détermination de la vitesse de ces météores par l'observation de la dérive horaire du radiant. Ref. 8281.

68: B. Kwal, Sur une possibilité d'interpréter les "bruits» radioélectriques du soleil et de la galaxie comme rayonnement des protons des radiations cosmiques dans les champs magnétiques intenses du soleil et des autres objets célestes. Ref. 12608.

69: J. - C. Pecker, Les moments nucléaires et la structure du noyau. Ref. 244.

70: M. Ma y ot, La prévision des nombres de Wolf et l'analyse linéaire. Ref. 6331.

71: R. Rigollet, Premiers résultats d'un programme de photométrie des petites planètes en rapport avec les séquences photovisuelles de l'Observatoire Leander McCormick. Ref. 7559.

72: R. Rigollet, L'essaim météorique des Iota Aquarides (Août) et son association probable avec la comète $1948 \mathrm{n}$. Ref. 8280 .

73: E. Schatzman, Les structures stellaires presque homologues. Ref. 5230.

74: E. Schatzman, Remarques sur le phénomène de nova (IV). L'onde de détonation due à l'isotope ${ }^{3} \mathrm{He}$. Ref. 11310.

75: E. Schatzman, Remarques sur le phénomène de nova (V). L'instabilité vibrationelle due à ${ }^{3} \mathrm{He}$. Ref. 11311.

76 : D. Barbier, Remarques sur l'équation intégrale de l'assombrissement au bord du soleil. Ref. 6402 .

268. Connaissance des Temps ou des mouvements célestes à l'usage des astronomes et des navigateurs pour l'an 1952, publiée par Le Bureau des Longitudes. Ref. 406.

269. Ephémérides Nautiques ou extrait de la Connaissance des Temps pour l'an 1952. Ref. 424.

\section{Poltawa}

270. Publikationen (Trudy) des Gravimetrischen Observatoriums Poltawa.

4: S. N. Aksentjewa, Endgültige Ergebnisse der Bestimmung der $\mathrm{M}_{2}$-Welle in den Schwereschwankungen in Tomask von 1912 bis 1920. Nicht referiert.

E. P. F jodorow, Über die Berücksichtigung des Einflusses der Meeresfluten bei der Untersuchung der luni-solaren Änderungen der Schwerkraft. Ref. 13113.

N. A. Popow, Über die kurzperiodischen Nutationsglieder in den Poltawaer Beobachtungen heller Zenitsterne. Ref. 3123.

E. W. Lawrentjewa, Beobachtungen der Zenitsterne $\alpha$ Persei und $\eta$ Ursae Majoris im Zeitraum 1944,6-1947,6. Ref. 13228.

N. A. Popow, Beobachtungen von $\alpha$ Persei und $\eta$ Ursae Majoris im Zeitraum 1947,6-1949,3. Ref. 13239.

W. K. Abold, Die Breite des Astronomischen Observatoriums Irkutsk aus 495 Beobachtungen von fundamentalen Zenitsternen in den fünf Jahren 1933-1937. Ref. 13201.

N. A. Popow, E. I. Jewtuschenko, Ableitung der Breitenschwankungen aus Beobachtungen am Universalinstrument. Ref. 13240.

E. P. Fjodorow, Das neue Programm für den Zeitdienst und seine Prüfung in Poltawa. Ref. 2314.

E. I. Jewtuschenko, I. P. Ogorodnik, Ergebnisse der Beobachtungen am großen Zenitteleskop des Observatoriums Poltawa im Zeitraum 1949,7-1950,9. Ref. 13221. 
S. G. Kulagin, Projekt des Programms von Breitenbeobachtungen für das Engelhardt-Observatorium. Ref. 13222.

E. P. Fjodorow, Untersuchung des Okularmikrometers des Wanschaffschen Vertikalkreises. Ref. 2222.

\section{Potsdam}

271. Mitteilungen des Astrophysikalischen Observatoriums Potsdam.

Nr. 32: W. Grotrian, H. Künzel, thber den Induktionsfluß durch Sonnenfleckengruppen der Klassen $\beta \gamma$ und $\gamma$. Ref. 6318.

\section{Poznań (Posen)}

272. Poznań Reprint.

Nr. 24: F. Koebcke, The radius of the earth's shadow from observations of lunar eclipses in the years 1924, 1927, 1931 and 1932. Ref. 7327.

25: F. Koebcke, Some remarks on Harzer's refraction tables. Ref. 3007.

26: F. Koebcke, Photographic observations of Jupiter's satellites. Ref. 7610.

27: S. Wierzbinski, Orbite provisoire de ADS $11468=$ A 1377. Ref. 10128.

\section{Pretoria}

273. Communications from the Radcliffe Observatory, Pretoria.

Nr. 17: D. S. Evans, The occultation of Antares of 1950 June 27-28. Ref. 9704.

\section{Princeton}

274. Contributions from the Princeton University Observatory.

Nr. 24: Im Druck.

25: R. S. Dugan, N. L. Pierce, F. B. Wood, Photometric researches. Twenty-four eclipsing variables. Ref. 11207.

275. Addenda to Newton Lacy Pierce's "A finding list for observers of eclipsing variables (Princeton Contribution No. 22)». By F. B. Wood. Ref. 11225.

\section{Pulkowo}

276. Publikationen (Trudy) des Astronomischen Hauptobservatoriums Pulkowo.

(2) 66: G. A. Tichow, Katalog der nach der Methode des Längsspektrographen erhaltenen Farben von Sternen in den Eichfeldern $44-91$. Ref. 9424.

67: R. S. Gnewyschewa, Katalog der Sonnentätigkeit 1948. Ref. 6353, 6660 .

68: G. K. Zimmerman, Ergebnisse der Beobachtungen am Vertikalkreis der Sternwarte Nikolajew in den Jahren 1929-1939. Ref. 9115.

277. Mitteilungen (Iswestija) des Astronomischen Hauptobservatoriums Pulkowo. 185 (146): A. N. Deutsch, Bahn und Masse des dunklen Begleiters des Doppelsterns 61 Cygni. Ref. 10108.

N. N. Pawlow, Das Meniskus-Passageninstrument. Ref. 2129. N. N. Pawlow, Die Neutralisation der Eingangskapazität der Elektrometerröhre. Ref. 2237.

N. N. Pawlow, Utber die Untersuchung der Zapfen tragbarer Passageninstrumente. Ref. 2509. 
A. A. Nemiro, Utber den Einfluß des Kollimationsfehlers auf die Ergebnisse von Rektaszensionsbeobachtungen. Ref. 2508.

N. W. Gamalej, Bestimmung genauer Örter der Kleinen Planeten Nemausa, Laetitia, Juno und Hebe. Ref. 7562.

W. W. Lawdowskij, Genaue Orter des Kleinen Planeten Juno (3) aus photographischen Aufnahmen im Jahre 1943. Ref. 7562. W. W. Lawdowskij, Genaue Orter des Kleinen Planeten Hebe (6) aus photographischen Aufnahmen im Jahre 1942. Ref. 7562. N. W. Fatschichin, Bestimmung der Orter des Kleinen Planeten Melpomene. Ref. 7562.

W. W. Lawdowskij, Der Einfluß der atmosphärischen Dispersion auf die Bestimmung genauer Orter Kleiner Planeten. Ref. 3120.

N. W. Fatschichin, Untersuchung des Pulkowoer Meßapparats Repsold I. Ref. 2503.

N.W. Gamalej, Untersuchung der Fehler des Meßapparates Repsold II. Ref. 2504.

6 (147): W. Krat, Ưber das Spektrum der Chromosphäre und schwacher Protuberanzen. (Aus dem Material der totalen Sonnenfinsternis vom 9. Juli 1945). Ref. 6204.

W. P. Wjasanizyn, Absolute Spektralphotometrie der Sonnenchromosphäre. Ref. 6203 .

O. A. Melnikow, B. K. Ioannissiani, Ein neues Teleskop mit spaltlosem Spektrographen für den ultravioletten Spektralbereich und seine Erprobung unter Hochgebirgsbedingungen. Ref. 2123. N. M. Goldberg-Rogosinskaja, Spektralphotometrische Untersuchung des Bedeckungsveränderlichen u Herculis. Ref. 11108. A. Sofronizkij, Über den Fehler bei der Ableitung der Bahnelemente der Bedeckungsveränderlichen für den Fall "KugelEllipsoid". Ref. 11134.

A. N. Dada jew, Ergänzungstabellen zur Ableitung der Elemente von Bedeckungssystemen mit verschiedenen Elliptizitäten der Komponenten. 1. (Totale Bedeckung). Ref. 11102.

\section{Richmond Hill}

278. Publications of the David Dunlap Observatory, University of Toronto.

1 24: H. B. Sawyer, Periods of variable stars in the globular cluster Messier 9. Ref. 12129.

25: J. F. Heard, The radial velocities and spectral features of twentyone Be stars with large rotational terms. Ref. 9212.

279. Communications from the David Dunlap Observatory.

Nr. 26: C. L. Seeger, R. E. Williamson, The pole of the galaxy as determined from measurements at $205 \mathrm{mc} / \mathrm{sec}$. Ref. 12627 .

27: R. E. Williamson, The atmosphere of the sun. Ref. 6018.

\section{Rio de Janeiro}

280. Anuário para o ano de 1951. Publicado pelo Observatório Nacional Rio de Janeiro. Ref. 447. Desgl. für 1952.

\section{Riverview}

281. Riverview College Observatory Publications.

26 (10): D. J. K. O'Connell, The so-called periastron effect in close eclipsing binaries. Ref. 11124.

D. J. K. O'Connell, New variable stars (fifth list). Ref. 11229. 
282. Riverview College Observatory. Reprint.

Nr. 3: D. J. K. O'Connell, A new eclipsing binary of very long period. Ref. 11123.

\section{Roms}

283. Osservatorio Astronomico Roma - Monte Mario - Gran Sasso. Contributi Scientifici.

Nr. 163: T. Fortini, L'assorbimento atmosferico della radiazione solare a Campo Imperatore, sul Gran Sasso d'Italia. Ref. 7256.

164: M. A. Giannuzzi, Sopra l'orbita della $\sigma$ Coronae Borealis. Ref. 10128.

165: G. Armellini Conti, Posizioni di pianeti e pianetini ed occultazioni lunari osservate nel 1950. Ref. 7340, 7401, 7562, 7601, 7701, 7801.

166: M. Cimino, T. Fortini, M. A. Giannuzzi, Area delle macchio solari durante l'anno 1950 , secondo i rilievi fotografici giornalieri effettuati all' Osservatorio Astronomico di Roma. Ref. 6353.

167: L. Gialanella, Sulla possibile natura e origine elettromagnetica delle pulsazioni osservate nel diametro del sole. Ref. 6004 .

168: M. A. Giannuzzi, Ricerca sopra l'assorbimento atmosferico della radiazione solare a Roma. Ref. 7258.

169: M. Cimino, A. Rainone, Una determinazione spettroscopica del periodo della rotazione solare nella regione verde dello spettro a diverse latitudini eliografiche. Ref. 6101.

170: M. Cimino, T. Fortini, Area, posizione eliografica e classifica della macchie solari durante il $1^{\circ}$ semestre 1951 , secondo i rilievi fotografici giornalieri dell' Osservatorio Astronomico di Roma. Ref. 6353.

\section{San Fernando}

284. Almanaque Nautico para el año 1952. Ref. 407.

285. Almanaque Nautico para uso de los navegantes para el año 1952. Ref. 425.

Sendai

286. Sendai Astronomiaj Raportoj.

212 (24): H. Nariai, On a new cosmological solution of Einstein's field equations of gravitation. Ref. 4142.

13 (25): K. Suda, On the scattering opacity in the envelope of Trumpler stars. Ref. 512l.

\section{Sonneberg}

287. Veröffentlichungen der Sternwarte in Sonneberg.

1 5: C. Hoffmeister, E. Rohlfs, P. Ahnert, Die veränderlichen Sterne der nördlichen Milchstraße. Teil VI. Ref. 11211.

288. Mitteilungen der Sternwarte zu Sonneberg.

Nr. 40: C. Hoffmeister, Beobachtungen von hochatmosphärischen Erhellungen des Nachthimmels in den Jahren 1943 bis 1945. Ref. 72155 .

289. Mitteilungen über veränderliche Sterne Nr. 129. Ref. 11229.

\section{Stockholm}

290. Stockholms Observatoriums Annaler.

16 4: T. Elvius, Catalogue of magnitudes, colours, spectral types, 
and spectrophotometric measurements for 2550 stars in Kapteyn's Selected Areas Nos. 2, 6, 7, 15, 16, 17, 18, 19, 20, 40, 41, and 42. Ref. 9412.

5: T. Elvius, A photometric and spectrophotometric investigation in twelve of Kapteyn's Selected Areas (Nos. 2, 6, 7, 15, 16, 17, $18,19,20,40,41$, and 42). Ref. 9413.

17 1: H. Kristenson, Spectrophotometric determinations of contact at total eclipses of the sun. Ref. 3208.

2: J. M. Ramberg, The distribution of light in the corona of the eclipse of 1914 August 21. Ref. 6201.

3: R. G. Langebartel, On the motion in barred spirals. Ref. 4411.

4: A. Elvius, A polarigraphic study of the spiral nebula NGC 5055 (M 63). Ref. 12414.

291. Stockholms Observatorium. Meddelande.

Nr. 66: A. Opolski, Orientation of the orbital planes of the visual binaries. Ref. 10115.

67: A. Opolski, Some remarks on the distribution of space velocities and kinetic energy on the Hertzsprung-Russell diagram. Ref. 12343.

68: B. Lindblad, The luminous surface and atmosphere of the sun. Ref. 6409.

69: B. Lindblad, The run of the spiral arms and the direction of rotation in NGC 4594. Ref. 12422.

70: B. Lindblad, Dynamical interpretation of velocity distribution and structural details in the galaxy. Ref. 12331, 12342.

71: Y. Ohman, On some analogous effects of light absorption in prominences and discharges. Ref. 6632.

72: B. Lindblad, An approach to the dynamics of stellar systems. Ref. 4413.

292. Stockholms Observatoriums Årsberättelse för året 1950. Ref. 1101.

\section{Strasbourg}

293. Annales de l'Observatoire de Strasbourg.

5 3: P. Muller, Mesures photométriques d'étoiles doubles. Ref. 10112. P. Muller, Magnitudes photométriques des composantes de 315 étoiles doubles ou multiples. Ref. 10113.

\section{Swarthmore}

294. Reprints of articles published by the Sproul Observatory.

Nr. 70: L. Binnendijk, A determination of the parallax and massratio of Furuhjelm 46 from photographs taken with the 24-inch Sproul refractor. Ref. 10127.

71: S. L. Lippincott, Parallax and orbit analysis of Ross 614 . Ref. 10128.

72: P. van de Kamp, S. M. Smith, A. Thomas, Parallax and orbital motion of Algol from photographs taken with the 24-inch Sproul refractor. Ref. 11115.

73: P. van de Kamp, Long-focus photographic astrometry. Ref. 10006.

74: P. van de Kamp, S. L. Lippincott, Flare-up of Krüger $60 \mathrm{~B}$. Ref. 11175.

75: H. Roth, The parallax of ADS 61 from photographs taken with the 24-inch Sproul refractor. Ref. 9307.

76: H. Roth, Parallaxes and proper motions of Wolf 294, Lalande 25372, and $\sigma$ Draconis. Ref. 9307. 


\section{Sydney}

295. Sydney Observatory. Astrographic Catalogue 1900.0.

29: Sydney Section R. A. $0^{\text {h }}$ to $6^{\text {h }}$, Dec. $-58^{\circ}$ to $-60^{\circ}$. Ref. 9120 .

32: Sydney Section R. A. $18^{\mathrm{h}}$ to $24^{\mathrm{h}}$, Dec. $-58^{\circ}$ to $-60^{\circ}$. Ref. 9120 .

296. Sydney Observatory Papers.

Nr. 12: W. H. Robertson, Occultations observed at Sydney Observatory during 1949. Ref. 7341.

13: H. Wood, Astronomy in Australia. Ref. 1165.

14: H. Wood, Tables for nearly parabolic elliptic motion. Ref. 4524.

15: H. Wood, Tables for hyperbolic motion. Ref. 4525.

16: H. Wood, Five figure tables for the calculation of ephemerides in parabolic and nearly parabolic motion. Ref. 4526.

297. Commonwealth Scientific and Industrial Research Organization, Australia, Division of Radiophysics.

J. P. Wild, Observations of the spectrum of high-intensity solar radiation at metre wavelength. IV. Enhanced radiation. Ref. 6555. W.N.Christiansen, J. V. Hindman, A. G. Little, R. PayneScott, D. E. Yabsley, C. W. Allen, Radio observations of two large solar disturbances. Ref. 6531.

J. H. Piddington, H. C. Minnett, Solar radio-frequency emission from localized regions at very high temperatures. Ref. 6549 . B. Y. Mills, A. B. Thomas, Observations of the source of radio-frequency radiation in the constellation of Cygnus. Ref. 12616.

F. J. Kerr, C. A. Shain, Moon echoes and transmission through the ionosphere. Ref. 7275 .

W. N. Christiansen, J. V. Hindman, A long-period change in radio-frequency radiation from the "quiet" sun at decimetre wave-lengths. Ref. 6532.

K. C. Westfold, The interpretation of the magneto-ionic theory. Ref. 262.

J. L. Pawsey, L. L. McCready, F. F. Gardner, Ionospheric thermal radiation at radio frequencies. Ref. 7280.

J. H. Piddington, The modes of formation of the ionospheric layers. Ref. 7281.

J. H. Piddington, H. C. Minnett, Observations of galactic radiation at frequencies of 1210 and $3000 \mathrm{Mc} / \mathrm{s}$. Ref. 12619 .

J. G. Bolton, K. C. Westfold, Galactic radiation at radio frequencies. IV. The distribution of radio stars in the galaxy. Ref. 12602.

A. G. Little, R. Payne-Scott, The position and movement on the solar disk of sources of radiation at a frequency of $97 \mathrm{Mc} / \mathrm{s}$. I. Equipment. Ref. 2118.

R. Payne-Scott, A. G. Little, The position and movement on the solar disk of sources of radiation at a frequency of $97 \mathrm{Mc} / \mathrm{s}$. II. Noise storms. Ref. 6548 .

J.H.Piddington, The origin of galactic radio-frequency radiation. Ref. 12620.

C. A. Shain, Galactic radiation at $18.3 \mathrm{Mc} / \mathrm{s}$. Ref. 12630 .

\section{Tasehkent}

298. Zirkular des Astronomischen Observatoriums Taschkent Nr. 238-249. $\S 23,63,66$. 


\section{Tokyo}

299. Nautical Almanac 1951, 1952. Ref. 408.

300. Japanese Ephemeris 1952. Ref. 409.

301. Abridged Nautical Almanac 1951, 1952. Ref. 426.

302. Annals of the Astronomical Observatory, University of Tokyo. Second Series.

3 2: K. Osawa, Distribution of the brightness at the extreme limb of the sun observed at the solar eclipse on May 9, 1948. Ref. 6206.

303. Tokyo Astronomical Bulletin. Second Series.

Nr. 32: R. Manabe, Occultation observations in Japan during 1948. Ref. 7341.

H. Hirose, S. Kaho, Photographic observations made with the Brashear astrograph (22). Ref. 7562, 7813.

33: M. Huruhata, T. Nakamura, Photoelectric determinations of the minima of eclipsing variables. Ref. 11229.

34: S. Nakano, H. Yasuda, R. Fukaya, Meridian observations of the right ascensions of minor planets. Ref. 7562 .

35: M. Notuki, On the coronal observation in Japan. Ref. 6712.

36: M. Notuki, S. Nagasawa, I. Shimizu, On a photometer for the observation of the coronal emission line. Ref. 2234.

37: M. Notuki, T. Ohe, M. Miyazawa, Dark filaments associated with solar eruptions. Ref. 6630.

38: S. Nakano, H. Yasuda, R. Fukaya, Meridian observations of the right ascensions of the sun, the moon and planets during the year 1950. Ref. 6001, 7101, 7113, 7301, 7401, 7601, 7701, 7801, 7804 .

39: H. Hirose, R. Manabe, Observations of the contact times of the solar eclipse of September 12, 1950. Ref. 6207.

H. Hirose, K. Tomita, Photographic observations made with the Brashear astrograph (26). Ref. 7562, 7813, §81.

40: H. Hirose, K. Tomita, Photographic observations made with the Brashear astrograph (27). Ref. 7562, 7813.

K. Tomita, Photographic observations of comets made with the Brashear astrograph. §81.

$K$. Tomita, Visual observations of comet 19481 made with the guiding telescope of the Brashear astrograph. Ref. 8164 . Discovery positions of Honda's comets. § 81 .

41: M. Utida, S. Habara, Y. Otoguro, F. Arata, Occultation observations in Japan during 1949. Ref. 7341.

304. Tokyo Astronomical Observatory Reprints.

Nr.61: K. Osawa, Photoelectric observation of the occultation of $\alpha$ Virginis (Spica) by the moon. Ref. 7339.

62: M. Miyadi, A note on the variation of international longitude. Ref. 13233.

63: H. Hirose, Summarised results of photographic and visual observations of the annular eclipse of May $8-9,1948$. Ref. 6206.

64: $K$. Tuzi, On the Tokyo-Mitaka catalogue of zenith stars and its catalogue difference. Ref. 9114.

65-71: Vgl. AJB 50.

72: N. Sekiguchi, Motions of the rotation axis of the elastic earth relative to the earth's body and to the space. Vgl. AJB 50 Ref. 13119.

73: S. Hata, On the signal-to-noise ratio of the multiplier phototube 1 P 21. Vgl. AJB 50 Ref. 2214.

74: T. Ura, T. Takenouchi, On special perturbations by the variation of elements. Ref. 4228. 
75: Z. Suemoto, Observation of the limb effect of faint Fraunhofer lines. Ref. 6426.

76: Z. Suemoto, Line contours in the anomalous regions of the sun. Ref. 6427.

77: S. Obi, Multiplet intensities for the lines ${ }^{1 D}-{ }^{3} \mathrm{P}$ of $\mathrm{O}$ III and $\mathrm{Ne}$ V. Ref. 241.

78: M. Huruhata, Photoelectric study of the zodiacal light. Ref. 8404.

79: H. Hirose, Preliminary report on contact observations. Ref. 6207.

T. Hatanaka, S. Suzuki, F. Moriyama, Preliminary report on the observation of the solar radio noise at the partial eclipse on September 12, 1950 . Ref. 6207.

80: T. Hatanaka, Y. Sekido, Y. Miyazaki, M. Wada, Solar radio outburst and increase of cosmic-ray intensities on September 20, 1950. Ref. 6539 .

81: Z. Suemoto, On the limb effect of faint Fraunhofer lines. Ref. 6428.

82: Z. Suemoto, On line contours in the anomalous regions of the sun. Ref. 6429.

83: H. Hirose, The effect of the terrestrial locality on the occultation of the stars by the moon. Ref. 7338 .

84: H. Hirose, Preliminary consideration on the locality effect of equinox corrections. Part I. Ref. 3115.

85: H. Hirose, Preliminary consideration on the locality effect of equinox correction. Part II. Ref. 3116.

86: N. Sekiguchi, Effects of the short period oceanic tides on the rotation of the earth. Ref. 13132.

87: T. Dambara, Photoelectric transit instrument. Ref. 2114.

88: F. Moriyama, On sunspots and magnetic storms. Ref. 6819.

89: M. Huruhata, Photoelectric studies of the night sky light (III). Ref. 72100.

90: T. Dambara, On the skewness of the velocity curves of the Cepheid variables. Ref. 11144.

91: N. Sekiguchi, Effects of the long period tides on the rotation of the earth. Ref. 13133.

92: Z. Suemoto, Electron temperature of the chromospheric eruption. Ref. 6647.

93: T. Dambara, On the phase differences measured in the sixcolor photometry of Cepheid variables. Ref. 11145 .

305. Tokyo Astronomical Observatory. Bulletin of Solar Phenomena.

2 4: October-December, 1950. Ref. 6353, 6556, 6660.

3 1-3: January-September, 1951, Ref. 6353, 6556, 6660 .

\section{Tortosa}

306. Boletín del Observatorio del Ebro.

86: Heliofísica 1948. Ref. 6353.

Toruń (Thorn)

307. Bulletin of the Astronomical Observatory of N. Copernicus University in Toruń.

Nr. 10: W. Dziewulski, The motions of the high velocity stars. Part I, Ref. 12837.

W. Dziewulski, C. Eubieńska, Visual observations of the long-period variable star $\chi$ Cygni. Ref. 11229. 
W. Dziew ulski, Visual observations of the long-period variable star o Ceti. Ref. 11229.

K. Serkowski, Minima of the eclipsing variable SW Lacertae. Ref. 11229.

R. Piatkowska, Visual observations of the variable star TT Herculis. Ref. 11229.

W. Dziewulski, Visual observations of the variable star SU Draconis. Ref. 11229.

W. Dziewulski, Visual observations of the variable star SW Draconis. Ref. 11229.

A. Lisicki, A new variable in the Pleiades. Ref. 11231.

\section{Trieste}

308. Osservatorio Astronomico di Trieste.

Nr. 243: E. L. Martin, La costellazione del Cancro. Ref. 9004.

244: G. B. Lacchini, Osservazioni di stelle variabili fatte nel 1950. Ref. 11216.

245: Annuario Astronomico per l'anno bisestile 1952 con le carte del cielo. Ref. 449.

246: E. L. Martin, Le congruenze settimanali del calendario gregoriano. Ref. 3307.

247: G. Romano, Osservazioni fotografiche e studio della Cefeide $\mathrm{X}$ Cygni. Ref. 11229.

\section{Turku}

309. Astronomia Observatorio de Universitato de Turku. Informo.

Nr. 7: Y.Väisälä, L. Oterma, Formulae and directions for computing the orbits of minor planets and comets. Ref. 4519.

8: L. Oterma, List of the photographs taken at the University Observatory of Turku during the period 1938-1949. Ref. 1243.

Uecle

310. Annales de l'Observatoire Royal de Belgique. Troisième Série.

5 4: S. Arend, L'amélioration de l'orbite d'une étoile double visuelle par application empirique de la méthode de la variation des éléments. Ref. 10102.

S. Arend, Orbite de l'étoile double visuelle ADS $11479=\mathrm{DBS}$ $8662=O \Sigma 359$. Ref. 10128 .

J.Dommange t, Méthode empirique systématique d'amélioration des éléments orbitaux d'une étoile double visuelle. Ref. 10109. J. Dommanget, Orbite de l'étoile double visuelle ADS $9352=$ BDS $6972=$ Hu 575. Ref. 10128.

5: F. Moreau, G. Becq, L. de Clerck, Amélioration des déclinaisons de 1339 étoiles fondamentales (Liste de Backlund) précédée d'une détermination de la constante de réfraction. Ref. 9105.

311. Bulletin Astronomique de l'Observatoire Royal de Belgique, à Uccle.

4 5: S. Arend, F. Riga ux, Observations photographiques de petites planètes et de la comète Shajn (1949e) à l'astrographe double de $40 \mathrm{~cm}$. Ref. 7562, §81.

S. Arend, F. Riga ux, Observations photographiques de petites planètes, de Pluton et de la comète Minkowski (1950 b) à l'astrographe double de $40 \mathrm{~cm}$. Ref. 7562, 7813, § 81 . 
S. Arend, F. Riga ux, Observations photographiques de petites planètes, de Neptune et de la comète Pajdusakova (195la) à l'astrographe double de $40 \mathrm{~cm}$. Ref. 7562, 7804, §81.

Positions d'astéroïdes et de la comète Kozik-Peltier (1939a) mesurées ou remesurées à la demande d'astronomes intéressés. Ref. 7562, \& 81 .

S. Arend, Positions de l'étoile de Barnard. Ref. 9101.

S. Arend, F. Rigaux, Comète Arend-Rigaux (195lb). §81.

G. Roland, Planète troyenne Arend 1950 SA. Ref. 7562.

312. Communications de l'Observatoire Royal de Belgique.

Nr. 22: R. Coutrez, Méthodes actuelles de la dynamique stellaire appliquée aux problèmes de la voie lactée et des nébuleuses extragalactiques. Ref. 12001.

23: Vgl. AJB 50 Ref. 6411.

24: P. Bourgeois, Les mouvements spatiaux des étoiles dans l'étude de la galaxie. Ref. 12334.

25: P. Bourgeois, Rapport sur les travaux scientifiques effectués pendant l'année 1950. Ref. 1101.

26: G. Coutrez, Observations des taches solaires en 1950. Ref. 6353.

27: E. Vandekerk hove, Un astrographe à champ de $180^{\circ}$. Ref. 2145.

28: P. J. Melchior, A. Huaux, Sur le déplacement annuel par rapport au globe terrestre de l'axe de rotation de la terre, en relation avec une oscillation annuelle de l'axe principal instantané d'inertie de la terre. Ref. 13230.

P. J. Melchior, Sur une nouvelle méthode d'analyse du mouvement du pôle à la surface de la terre. Ref. 13231 .

29 : E. Vandekerkhove, Un télescope coudé de Schmidt. Ref. 2146.

30: E. Vandekerkhove, Etude d'une méthode pour déterminer des vitesses radiales avec un prisme-objectif. Ref. 2413.

E. Vandekerkhove, Détermination des paramètres de raies composantes dont on connaît l'effet résultant. Ref. 2414.

31: L. Neven, C. de Jager, The model of the solar atmosphere and the continous absorption coefficient in the infrared. Ref. 6119.

32: D. de Smet-de Potter, Magnitudes et indices de couleur d'étoiles aux environs du pôle. Ref. 9422.

33: M. Migeotte, L. Neven, Nouvelles raies de méthane tellurique. Ref. 7235.

34: M. Migeotte, L. Neven, L'activité de la mission scientifique belge à la station du Jungfraujoch en 1950 et 1951. Ref. 1216.

35: J. Hunaerts, Le problème de l'identification des radiations cométaires à partir de spectres synthétiques. Ref. 8012 .

313. Observatoire Royal de Belgique. Monographies.

Nr. 2: S. Arend, Théorie de l'équatorial visuel et de l'équatorial photographique. Réglage pratique de l'équatorial visuel et de l'astrographe. Ref. 2103.

\section{Upper Darby}

314. The Flower Astronomical Observatory. Reprint.

Nr. 80: S. G. Barton, New double stars. Ref. 10125.

\section{Uppsala}

315. Uppsala Astronomiska Observatoriums Annaler.

2 9: E. Lyttkens, Problems of dark nebulae, treated by the method of moments. Ref. 5307 . 
10: E. Bodén, A research on the galactic clusters NGC 436 and NGC 457. Ref. 12103.

3 1: Vgl. AJB 49 Ref. 6204.

2: Vgl. AJB 49 Ref. 2240.

3: Vgl. AJB 49 Ref. 12336.

4: E. Bodén, Photographic, photovisual and red magnitudes in the galactic cluster M 36. Ref. 12104.

5: Vgl. AJB 52.

6: B. Westerlund, Luminosity effects and colour-equivalents as measured in short stellar spectra. I. B, A, and F stars. Ref. 9561.

316. Uppsala Astronomiska Observatorium. Meddelande.

Nr. 104: N. V.E. Nordenmark, Laurentius Paulinus Gothus föreläsningar vid Uppsala universitet 1599 över Copernicus hypotes. Ref. 1442.

\section{Utrecht}

317. Recherches Astronomiques de l'Observatoire d'Utrecht.

12 1: W. J. Claas, The composition of the solar atmosphere. Ref. 6405.

2: Photometric Catalogue of Fraunhofer lines $\lambda 6600-\lambda 8770$. Ref. 6430.

\section{Victoria}

318. Publications of the Dominion Astrophysical Observatory Victoria, B. C.

8 12: A. B. Underhill, A model atmosphere for an early O-type star. Ref. 5128.

13: A. B. Underhill, On the luminosity effects of the hydrogen lines in O-type spectra. Ref. 9557.

14: J. L. Climenhaga, A. McKellar, R. M. Petrie, Spectrographic observations of Epsilon Ursae Minoris. Ref. 10202.

15: J. A. Pearce, R. M. Petrie, Revised radial velocities of seventynine B-type stars. Ref. 9214.

9 1: C. S. Beals, The spectra of the P Cygni stars. Ref. 11160.

319. Contributions from the Dominion Astrophysical Observatory Victoria, B. C.

Nr. 23: A. B. Underhill, An estimate of the relative helium content of the early-type stars. Ref. 5127.

\section{Washington}

320. The American Ephemeris and Nautical Almanac for the Year 1953. Ref. 410.

321. Astronomical Papers prepared for the use of the American Ephemeris and Nautical Almanac.

12: W. J. Eckert, D. Brouwer, G. M. Clemence, Coordinates of the five outer planets $1653-2060$. Ref. 7604 .

322. United States Naval Observatory Circular.

Nr. 25-26: L. T. Day, Positions, areas, and counts of sunspots OctoberNovember 1950. Ref. 6353.

27: Annular eclipse of September 1, 1951. Path of annular phase in the United States. Ref. 6209.

28-29: L. T. Day, Positions, areas, and counts of sunspots December 1950-February 1951. Ref. 6353.

30: W. Markowitz, Sunspot areas and numbers as indices of solar activity. Ref. 6329. 
31-33: L. T. Day, Positions, areas, and counts of sunspots March-July 1951. Ref. 6353.

323. Smithsonian Institution. Publication.

4027: B. Lindblad, The luminous surface and atmosphere of the sun. Ref. 6409.

4029: H. Brown, The composition of our universe. Ref. 5004.

\section{Wellington}

324. Carter Observatory Reprint.

Nr. 14: R. G. Dick, I. L. Thomsen, Geodetic positions of astronomical Observatories at Kelburn, Wellington. Ref. 13205.

I. L. Thomsen, Daylight fireball, 1951 February 4. Ref. 8296.

I. L. Thomsen, New Zealand determinations of relative sunspot numbers. Ref. 6346.

15: D. M. Garner, Preliminary investigation of aurora australis activity from 1931 to 1949 . Ref. 72119.

16: Martin Maxwell Fleming Luckie, O. B. E. Ref. 1001.

I. L. Thomsen, Large sunspots and the phase of sunspot activity. Ref. 6347.

17: I. L. Thomsen, The Abridged Nautical Almanac. Ref. 458.

325. Astronomical Bulletin.

Nr. 35: I. L. Thomsen, Report of the Carter Observatory Board for the year ended 1951, March 31. Ref. 1101.

36: Programme for public sessions, 1952. Ref. 1247.

Wien

326. Mitteilungen der Universitäts-Sternwarte Wien.

5 I: K. Ferrari d'Ochieppo, Anfangswachstum und Auflösung der Sonnenflecken I. Vgl. AJB 50 Ref. 6307.

2: H. Krumpholz, Beobachtungen von Kometen. $\$ 81$.

3: T. Widorn, Der Lichtwechsel des Saturnsatelliten Japetus im Jahre 1949. Vgl. AJB 50 Ref. 7707.

4: H. Haupt, țber Phasenkoeffizienten und Albedo der Kleinen Planeten Ceres, Pallas, Juno und Vesta. Vgl. AJB 50 Ref. 7549.

5: I. Nikoloff, Definitive Bahnbestimmung des Kometen 1936 III (Kaho-Kozik-Lis). Vgl. AJB 50 Ref. 8134.

6: H. Socher, Die Polhöhe der Universitäts-Sternwarte Wien. Vgl. AJB 50 Ref. 13225.

7: H. Socher, Veränderliche Fundamentalsterne der "Potsdamer Durchmusterung». Vgl. AJB 50 Ref. 11007.

8: H. Socher, Brechzahl und Refraktion der atmosphärischen Luft. Ref. 7246.

9: K. Ferrari d'Ochieppo, Anfangswachstum und Auflösung der Sonnenflecken III. Ref. 6312.

10: H. Eichhorn, Die Ausnahmefälle bei der Bestimmung einer Kreisbahn. Ref. 4506.

11: H. Eichhorn, Zur Erfassung der Ausnahmefälle bei der Bahnbestimmung. Ref. 4507.

12: H. Haupt, H. Auzinger, Helligkeitsbeobachtungen von Kleinen Planeten. Ref. 7557, 7805.

\section{Williams Bay}

327. T. Page, The Yerkes Collection of Astronomical Photographs. Ref. 1249. 
LVIII Übersicht über die referierten Zeitschriften

\section{Zürich}

328. Publikationen der Eidgenössischen Sternwarte Zürich.

9 5: M. Wald meier, Heliographische Karten der Photosphäre für das Jahr 1950. Ref. 6353.

329. Astronomische Mitteilungen der Eidgenössischen Sternwarte Zürich.

Nr. 173: M. Waldmeier, Spektralphotometrische Klassifikation der Protuberanzen. Ref. 6656.

174: M. Waldmeier, Die Form der monochromatischen Korona. Ref. 6723.

175: M. Waldmeier, Die neue Koronalinie 5445 A. Ref. 6724.

176: M. Waldmeier, Die Sonnenaktivität im Jahre 1950. Ref. 6353, $6660,6727$.

177: M. W ald meier, Katalog der grünen Koronastrahlen 1947-1949. Ref. 6727.

178: M. Waldmeier, Die Sonnenkorona vom 8. Juli 1851. Ref. 6725.

330. Quarterly Bulletin on Solar Activity Nr. 89-92. Ref. 6353, 6556, 6660, 6727.

331. Tätigkeitsbericht der Eidgenössischen Sternwarte für das Jahr 1950. Ref.110I. 